\title{
Design, Fabrication, and Test of a 5-kWh/100-kW Flywheel Energy Storage Utilizing a High-Temperature Superconducting Bearing
}

M. Strasik, P. E. Johnson, A. C. Day, J. Mittleider, M. D. Higgins, J. Edwards, J. R. Schindler, K. E. McCrary, C.R. Mclver, D. Carlson, J. F. Gonder, and J. R. Hull

November 15, 2007

University of Cambridge, UK

This work was partially supported by the U.S. Department of Energy, Offices of Electricity Delivery and Energy Reliability under the Cooperative Agreement DE-FC36-99G010825, Contract W-31-109-Eng-38, and Sandia National Laboratories Energy Storage Program Contract 24412 


\section{Outline}

- Flywheel application description

- 5 kWh /100 kW FES design and test results

- Previous HTS bearing and cryogenic set-up and results

- Direct cooled bearing design and test results

- Description of direct cooled test set-up

- Summary 


\section{Boeing Flywheel Facility - Seattle}

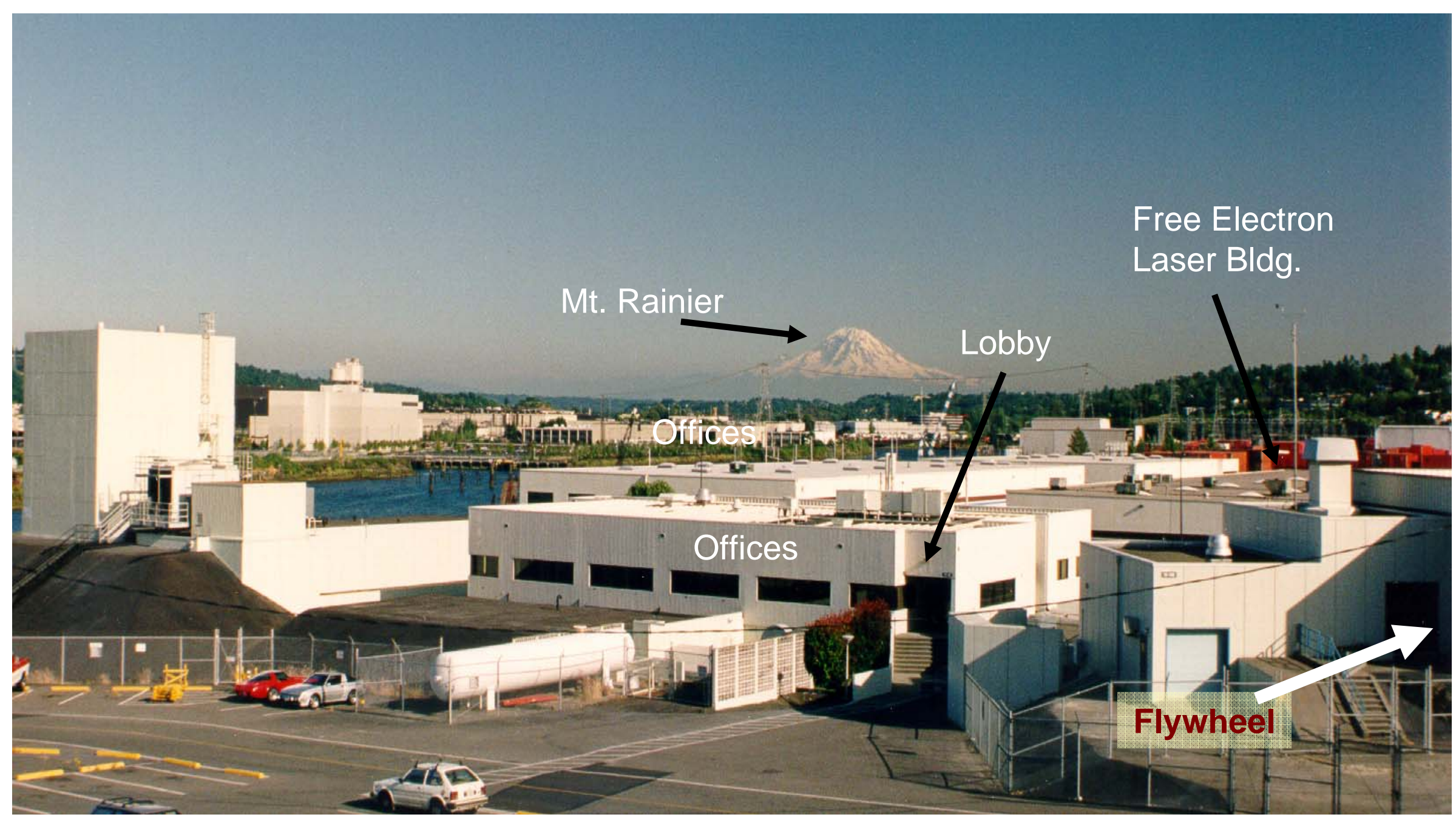




\section{BOEING South Park - Flywheel Integrated Spin Test Facility}

\section{5-08\& -11 Bidg}

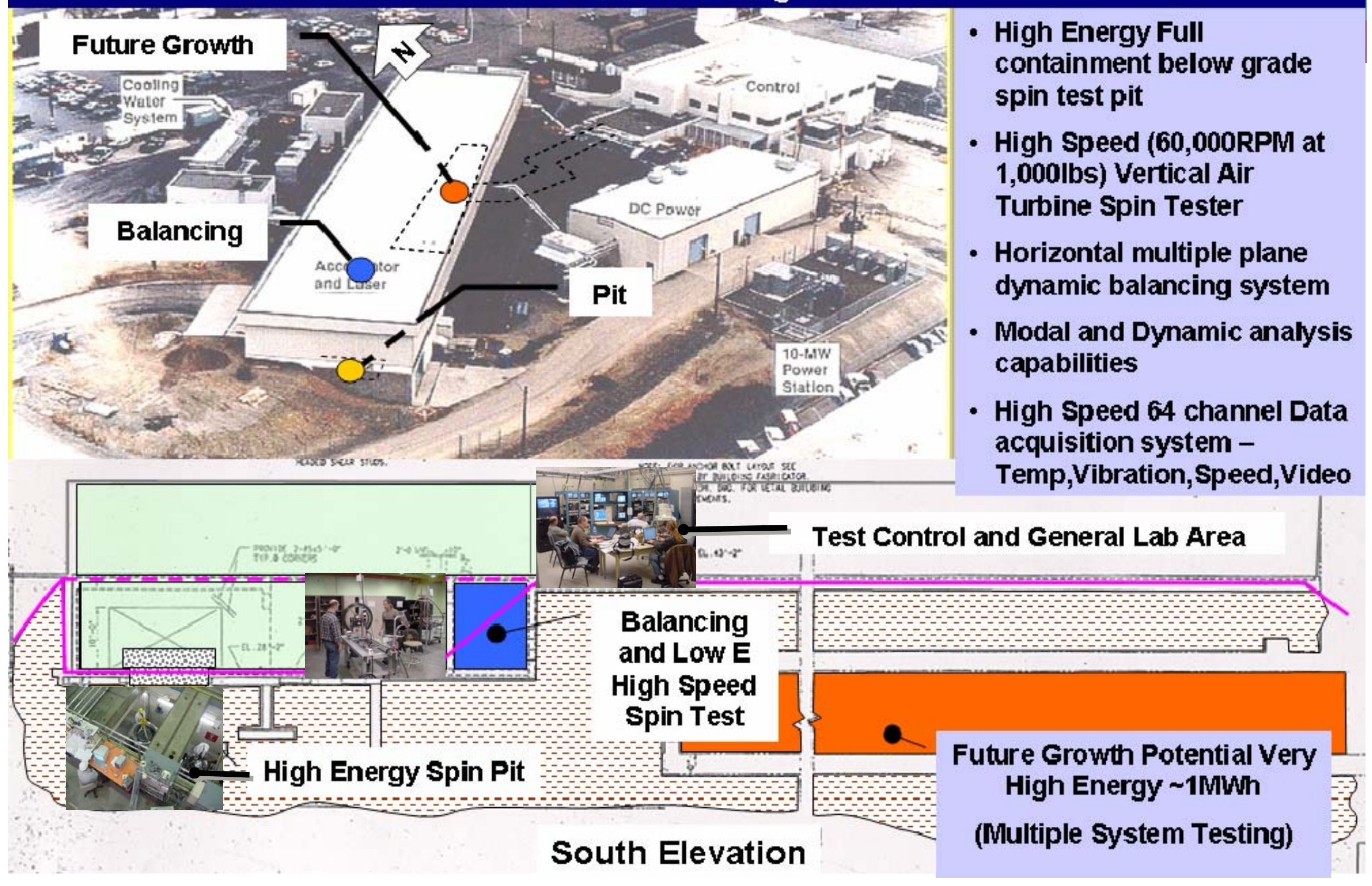




\section{Boeing Flywheel Spin Test Facility}

Boeing Technology | Phantom Works

Flywheel Energy Storage

Test pit with concrete blocks

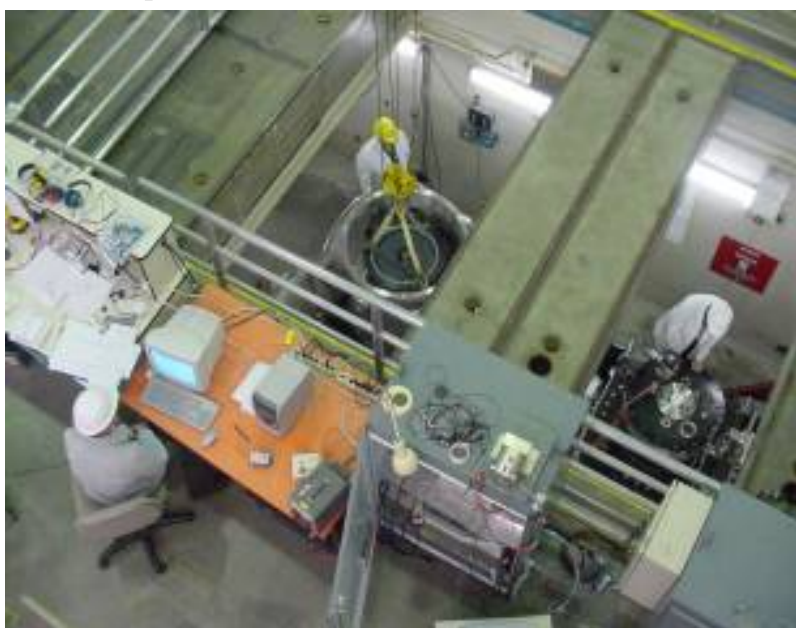

Flywheel test chamber

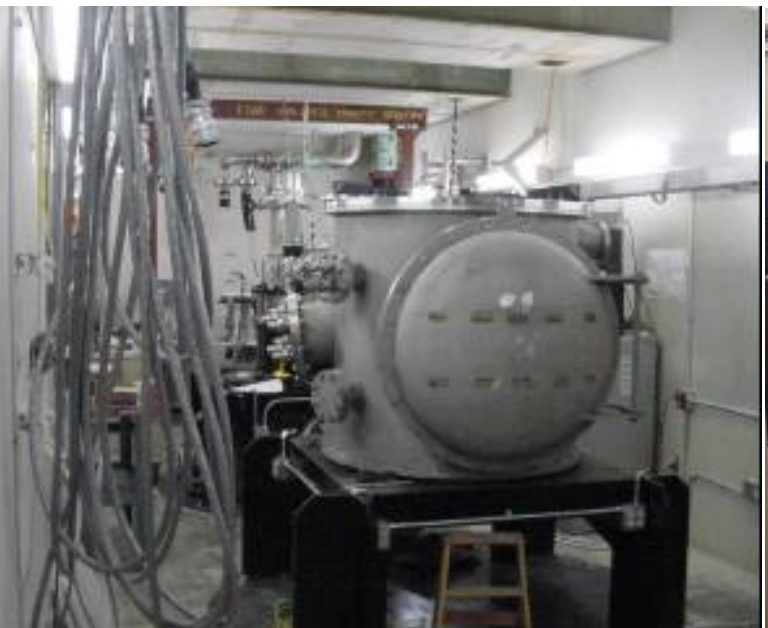

Control room

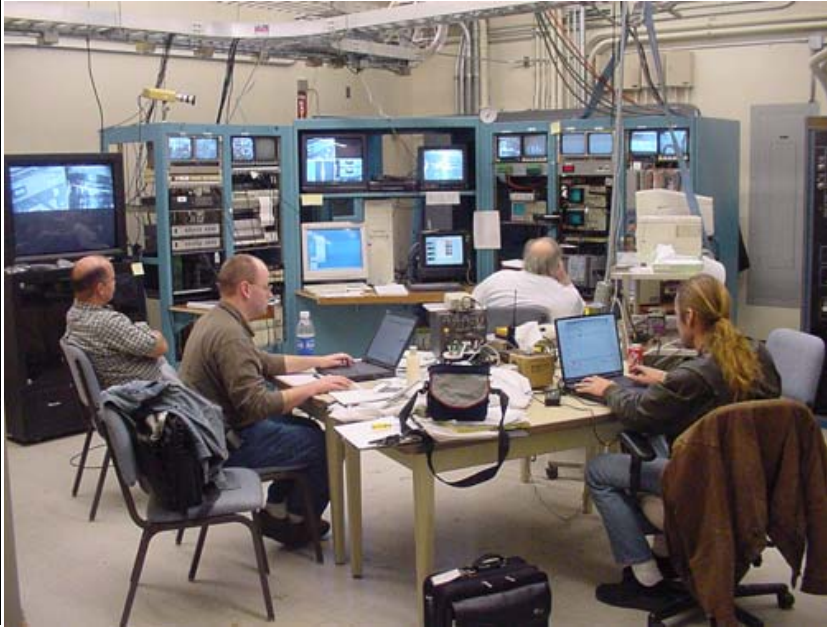

\section{Largest Flywheel Spin Test Facility on the West Coast}

$2^{\text {nd }}$ Level test area

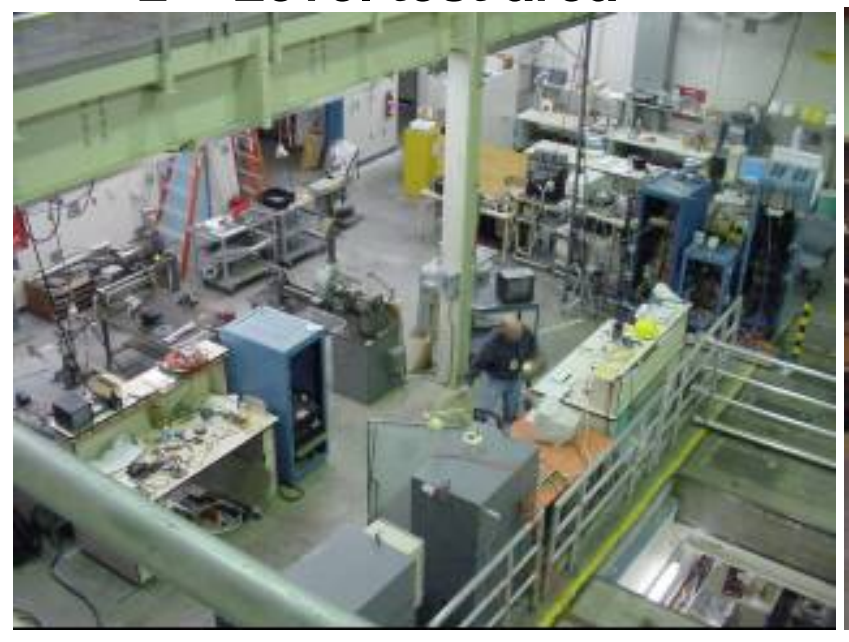

Balancing spin system

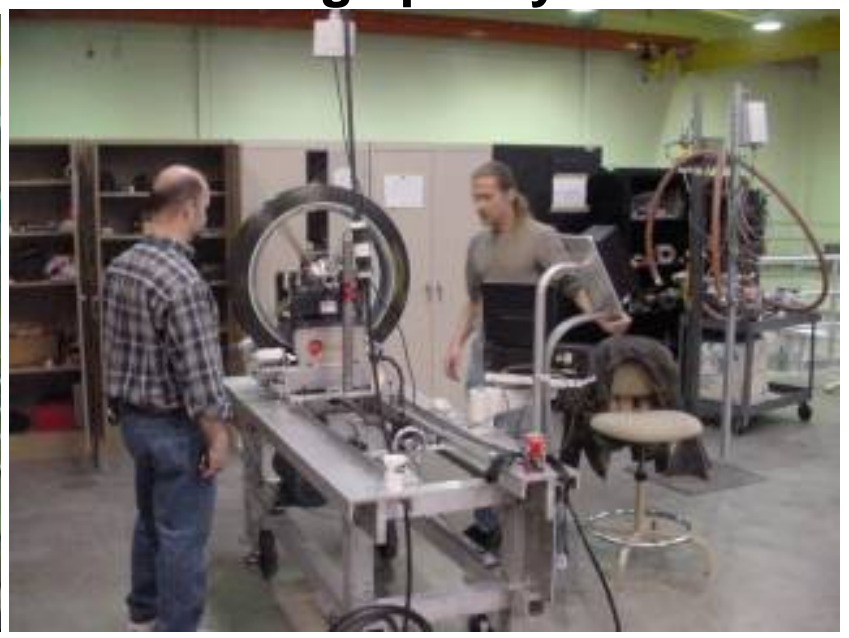

Flywheel test facility

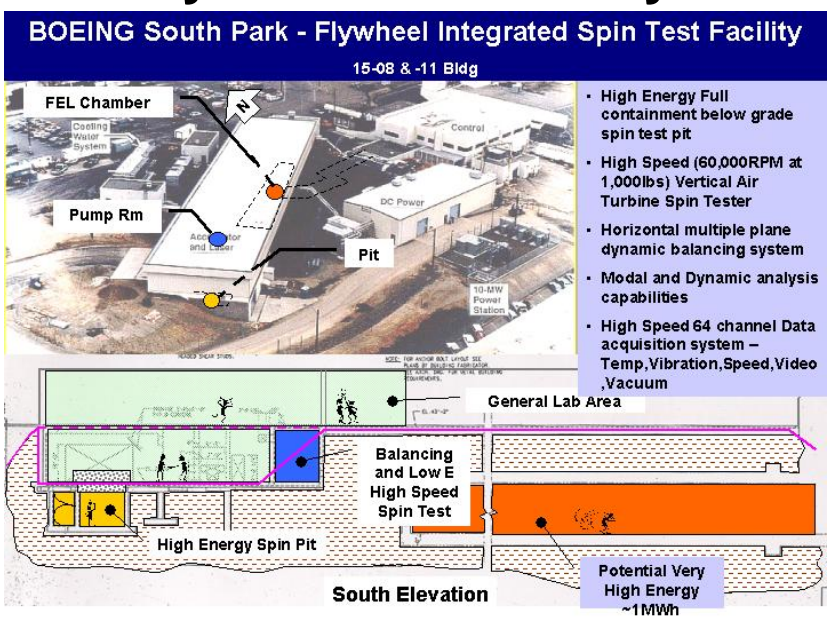




\section{Flywheel Electricity Systems}

Boeing Technology | Phantom Works

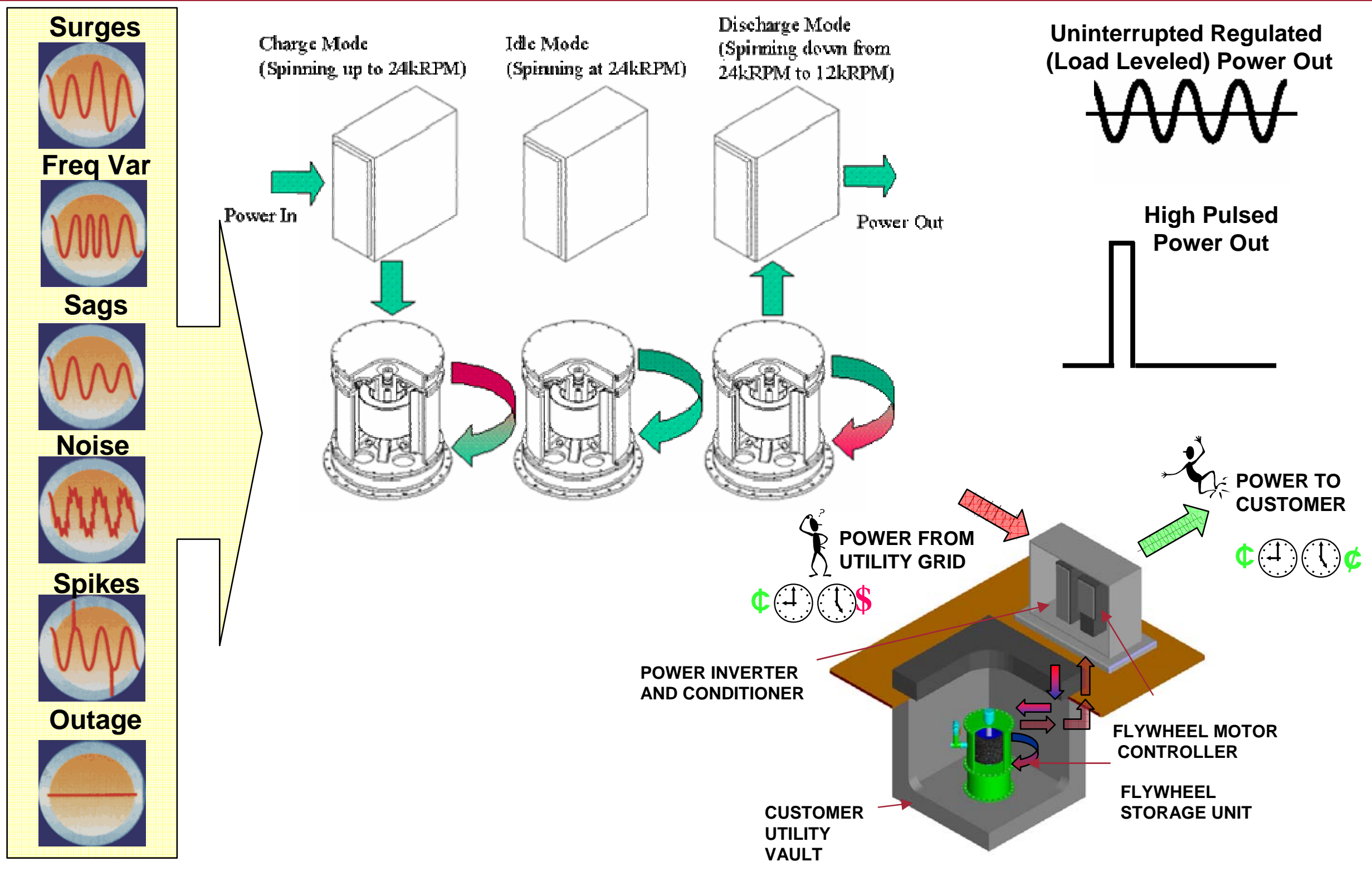




\section{Boeing Flywheel Development History}

Boeing Technology | Phantom Works

Flywheel Energy Storage

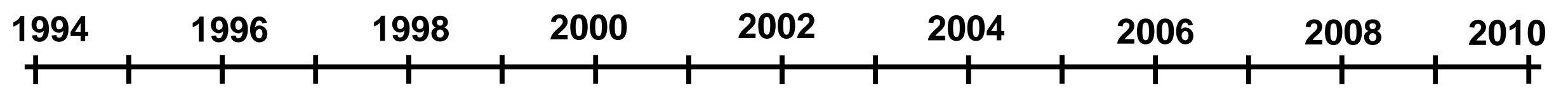

Design Concept 1 MWh FES for Commonwealth Edison
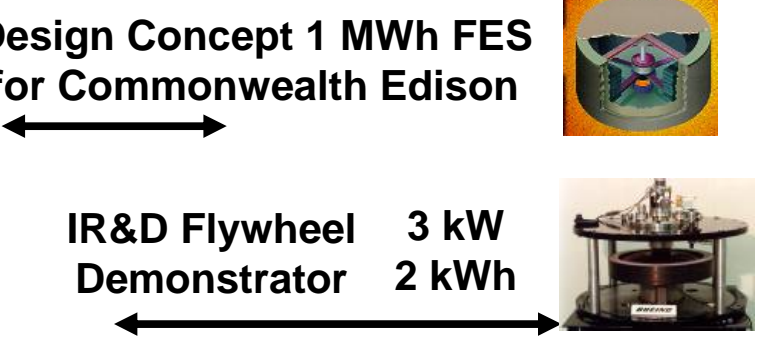

DOE Phase 1 and 2 Contract

(\$12M)
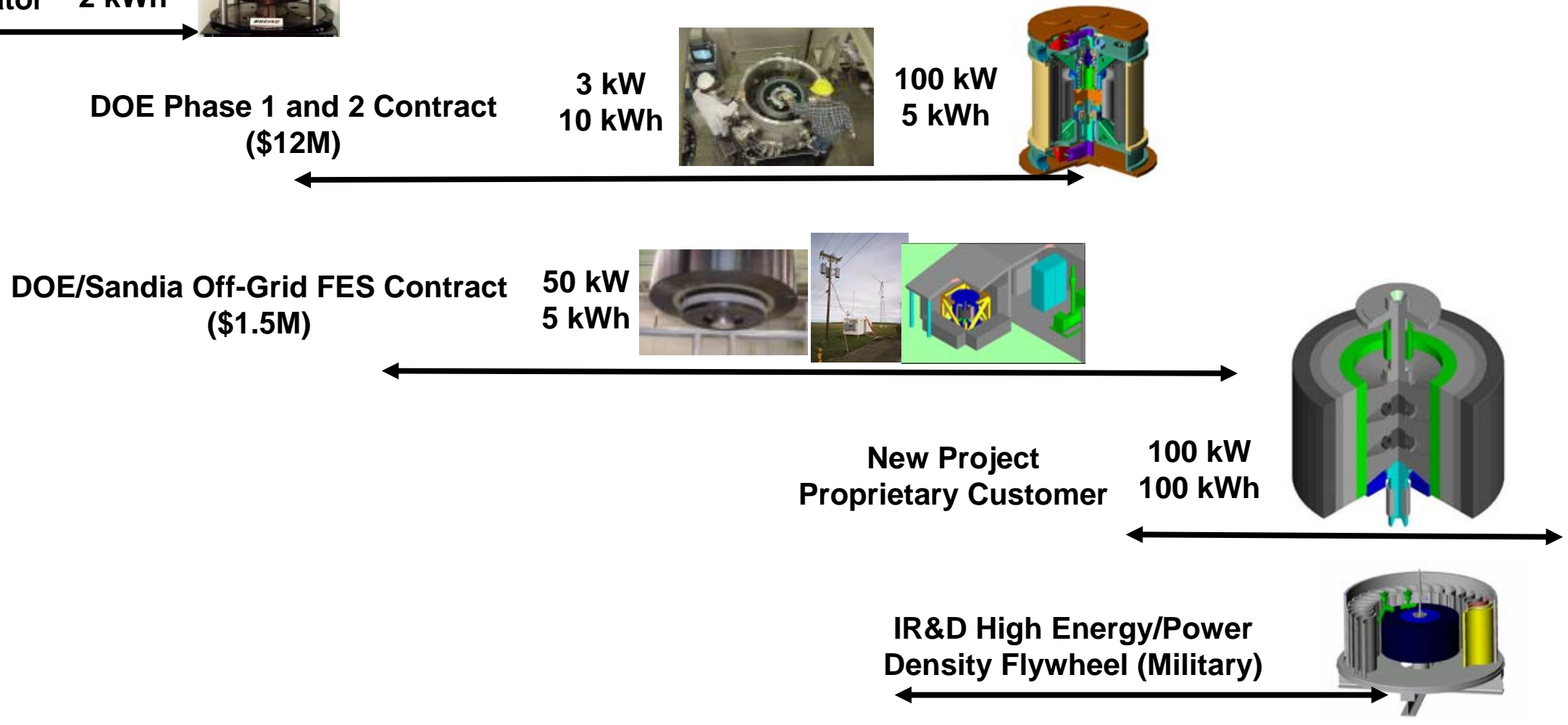


\section{Why Flywheels and Superconducting Bearings?}

- Why Pursue Flywheel Energy Storage?

- Non-toxic and low maintenance

- Potential for high power density (W/ kg) and high energy density (W-Hr/ kg)

- Fast charge / discharge times possible

- Cycle life times of $>25$ years

- Broad operating temperature range

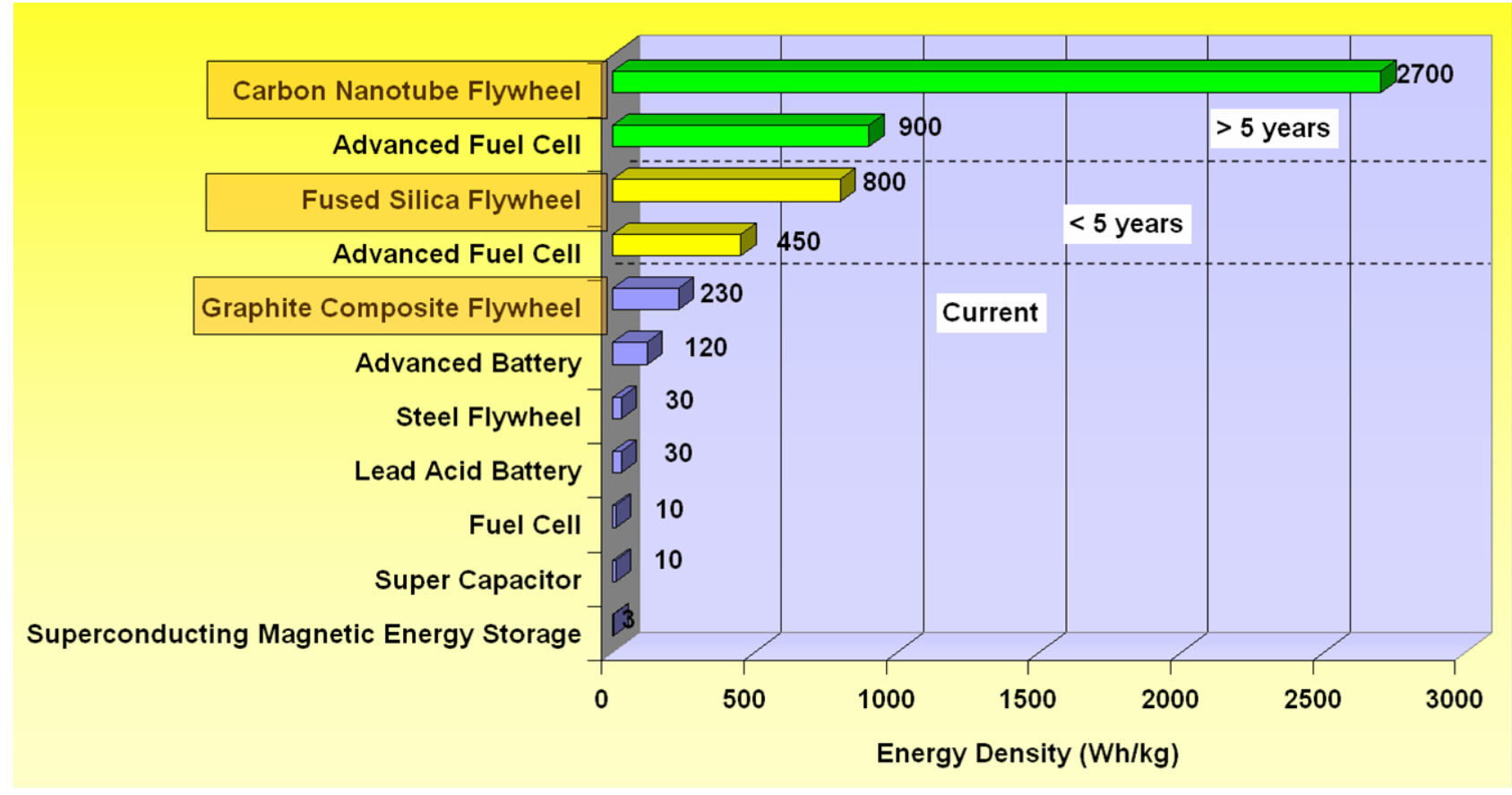

- Why use HTS bearings?

- Simple passive system

- Very low frictional loss

- Very long lifetime

- Low cost and maintenance

- Lower tolerance for balancing of dynamic structures

- High speed capability (> 500,000 RPM)

- Adjustable stiffness and damping

Boeing Superconducting Bearing Offers Many Design and Operational Benefits Over Conventional Bearing Systems 


\section{Boeing 100 kW / 5 kWh UPS Flywheel System Design}

Boeing Technology | Phantom Works

Flywheel Energy Storage

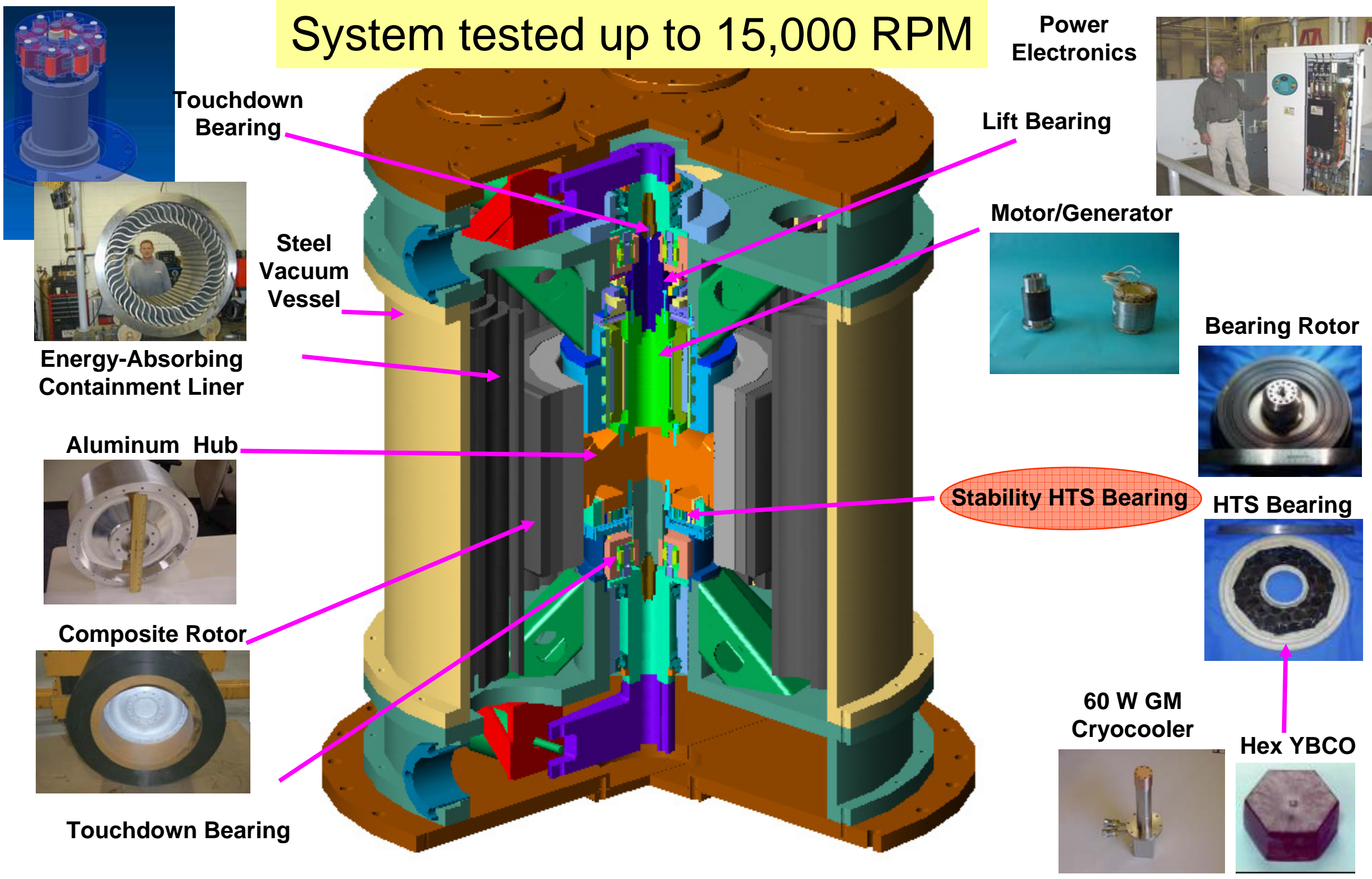




\section{Boeing Cryogenic Bearing Enables Low Loss}

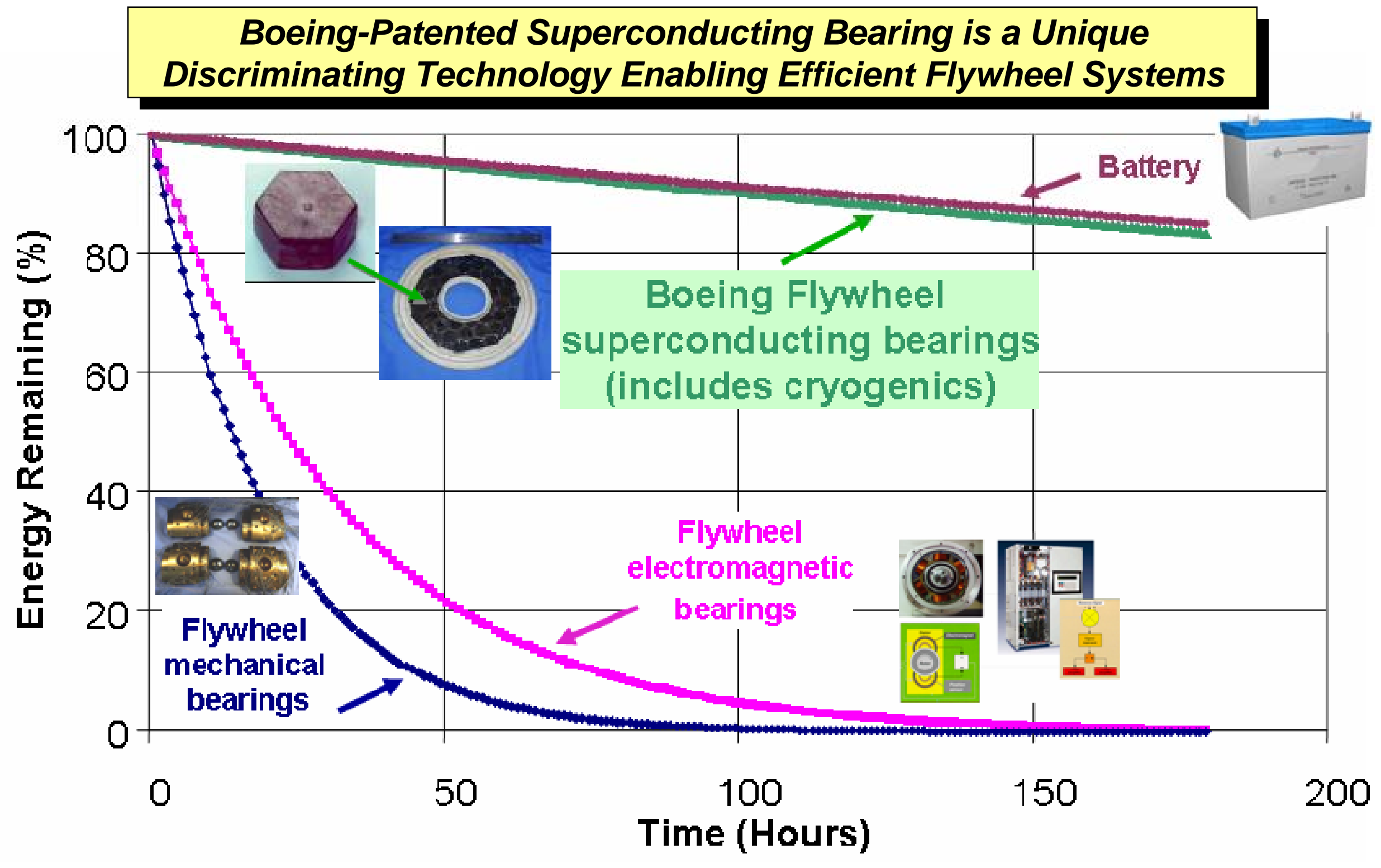




\section{Superconducting Bearing System}

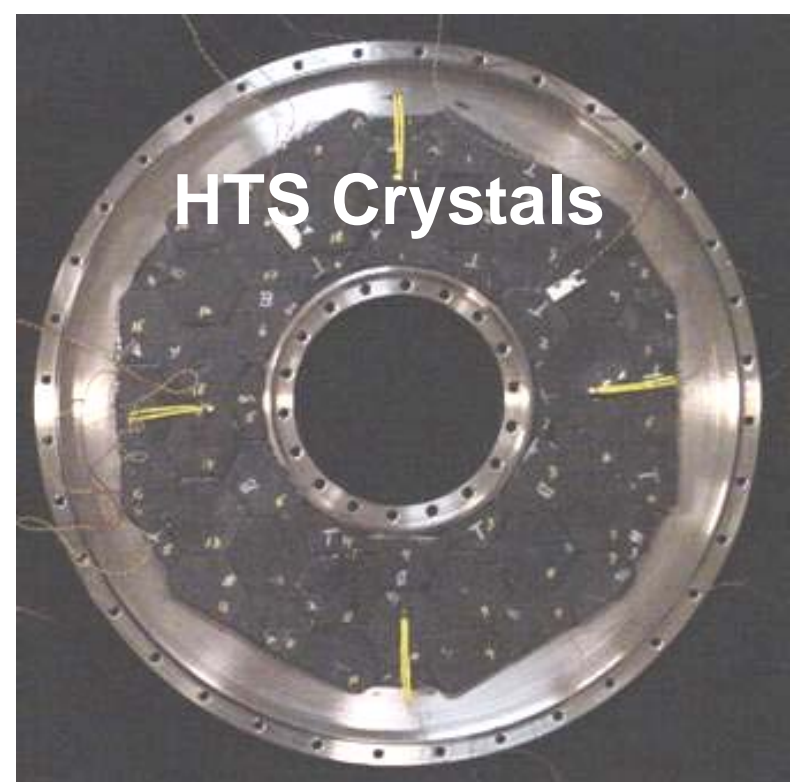

HTS Bearing Rotor

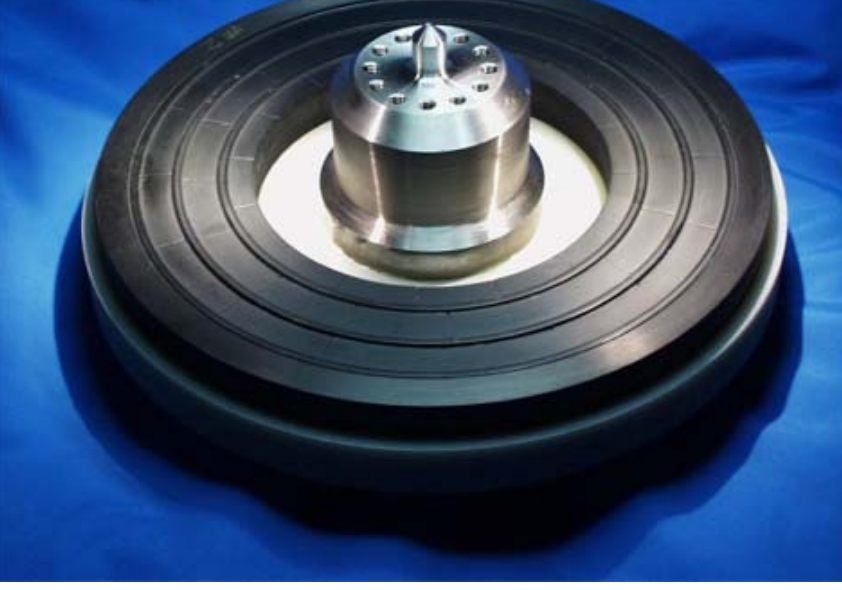

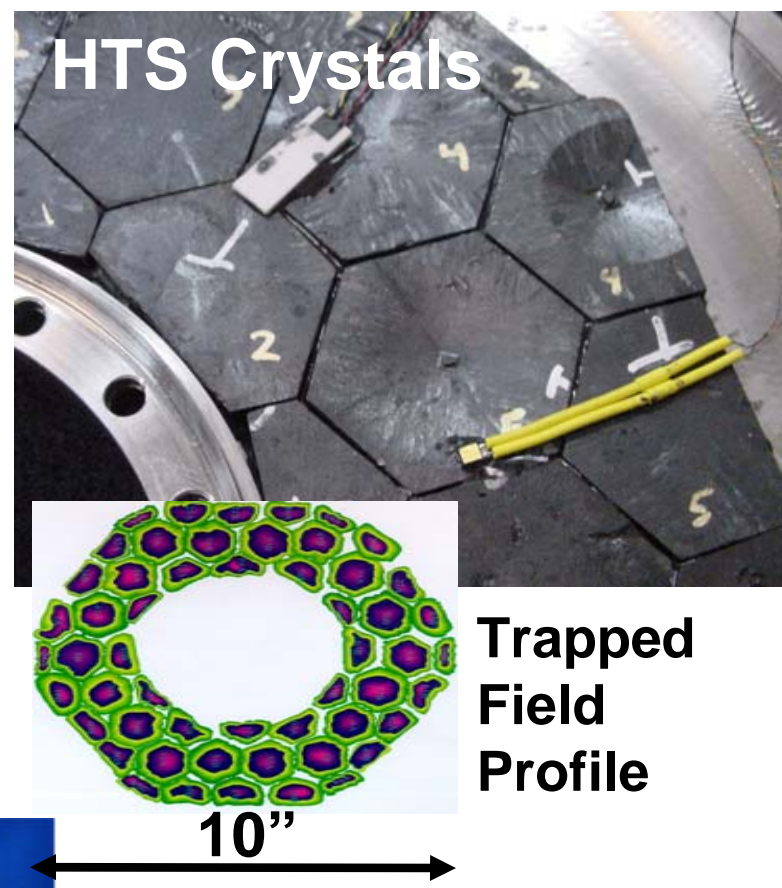

Structural Bearing Models

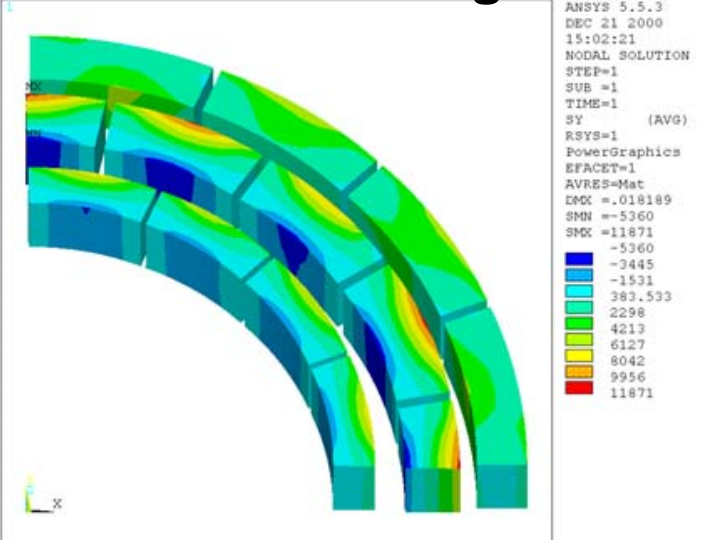

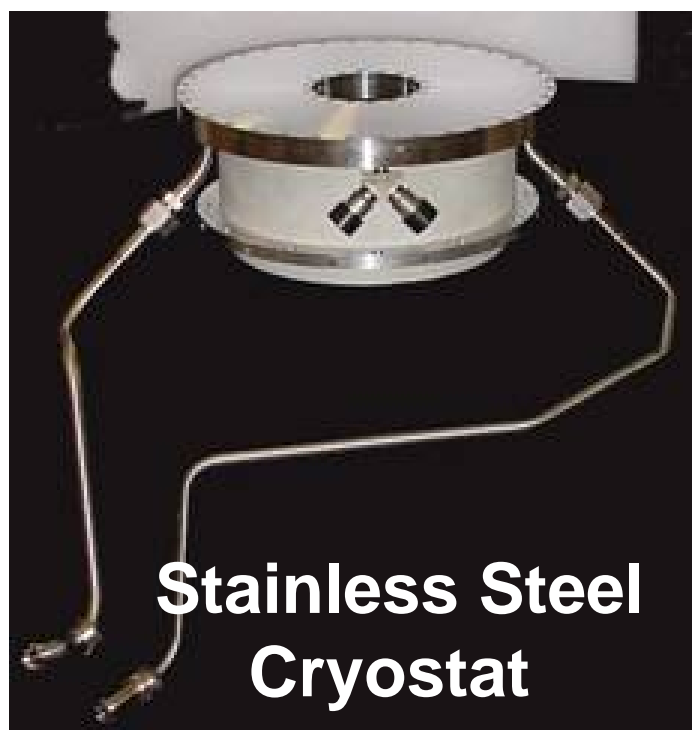

Electromagnetic Models

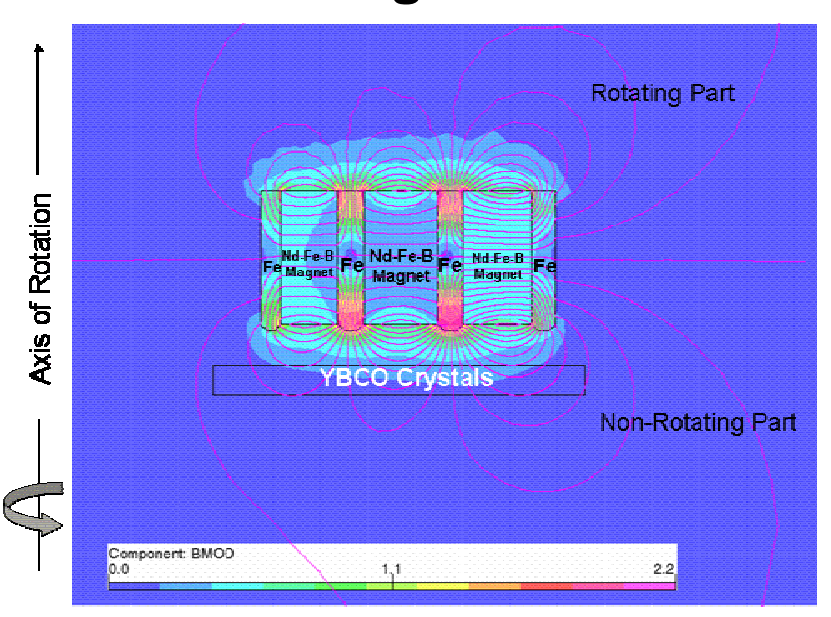




\section{Flywheel Rotor Assembly}

- The flywheel team has successfully tested a composite flywheel system weighing $360 \mathrm{lbs}$ and supported by HTS bearing up to 15,000 RPM

- Superconducting bearing performance confirmed estimate of $<0.2 \%$ per hour
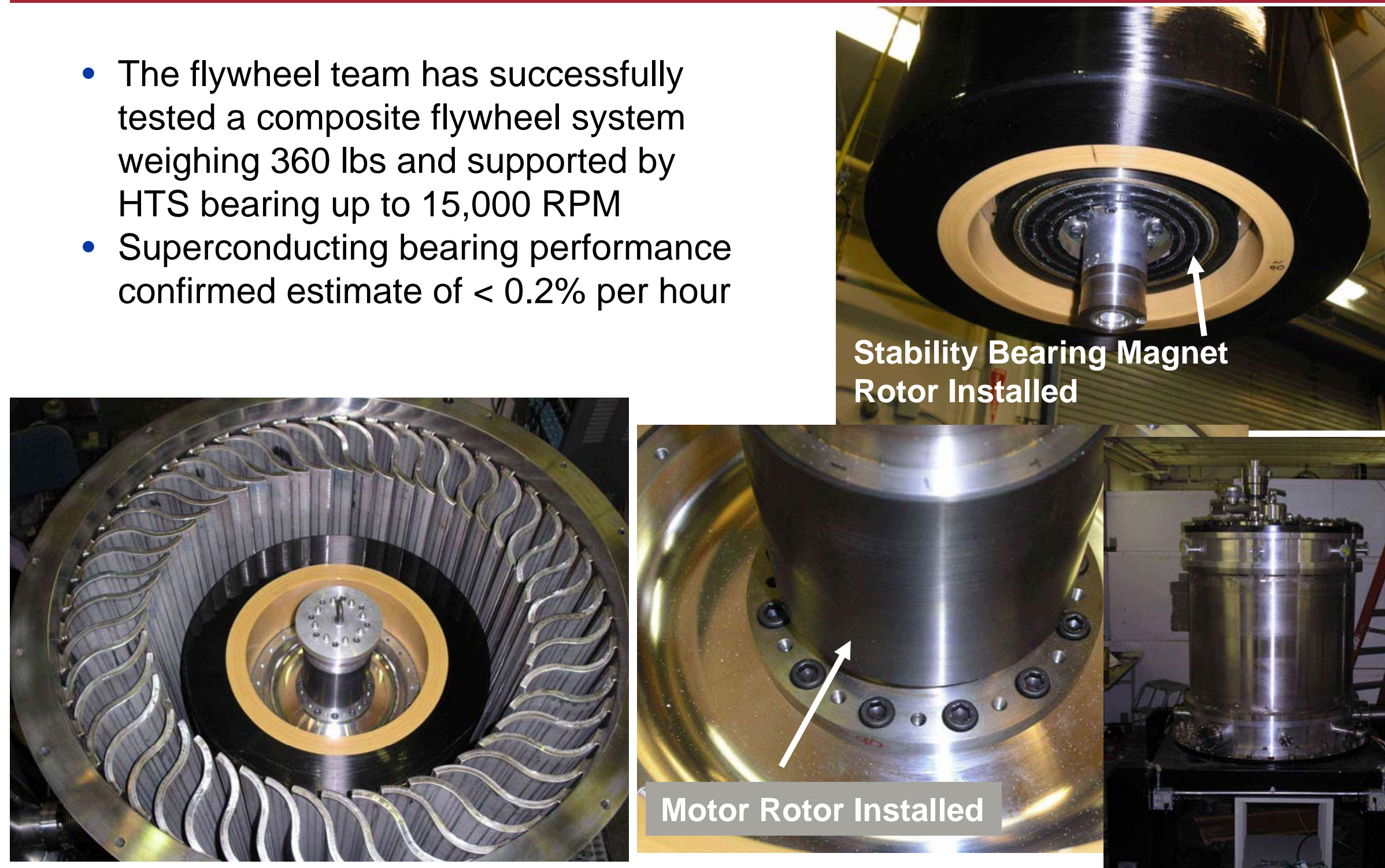

Stability Bearing Magnet

Rotor Installed

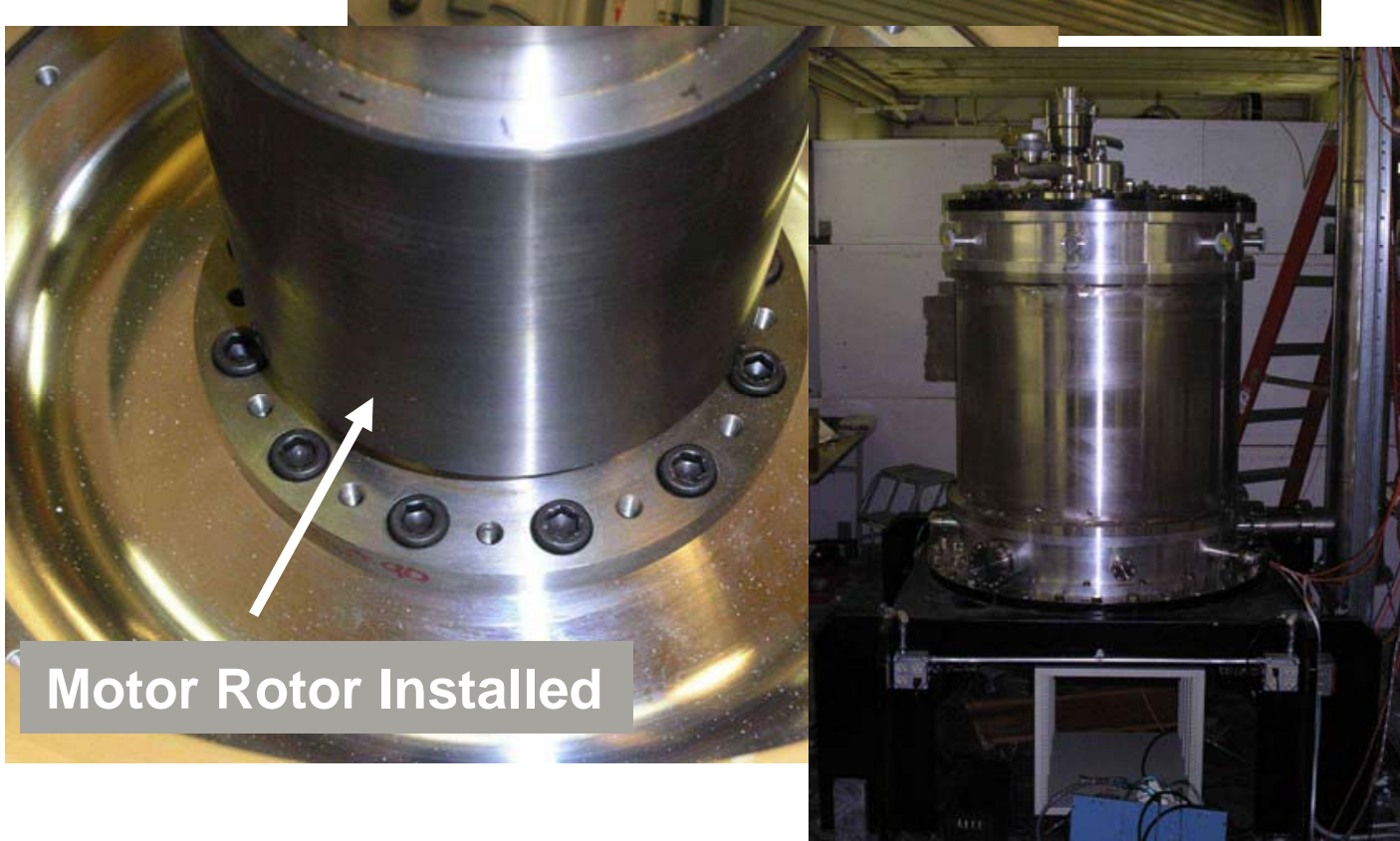




\section{Stability Bearing Rotor Installation}

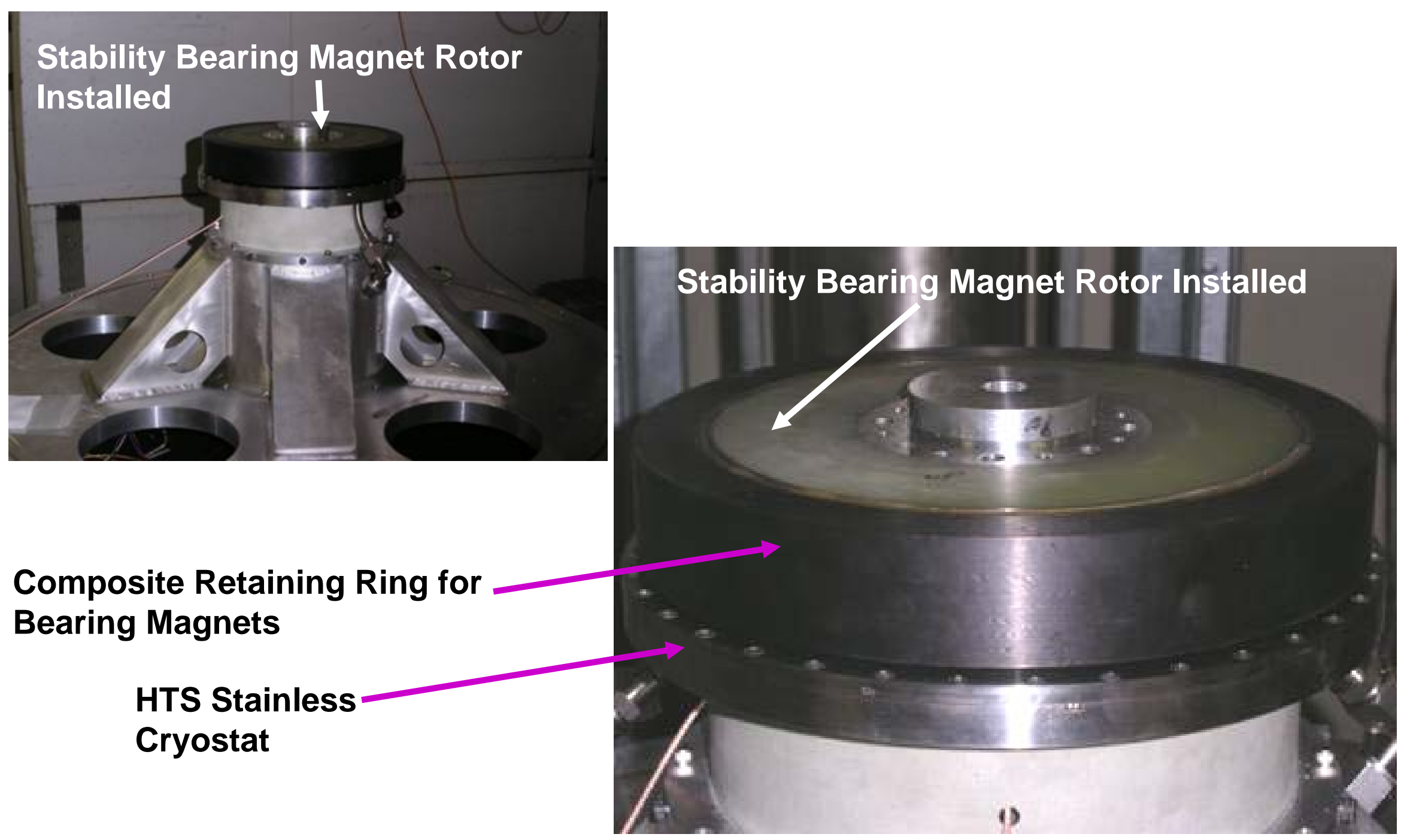




\section{Rotor Installation and Lift Magnet Assembly}

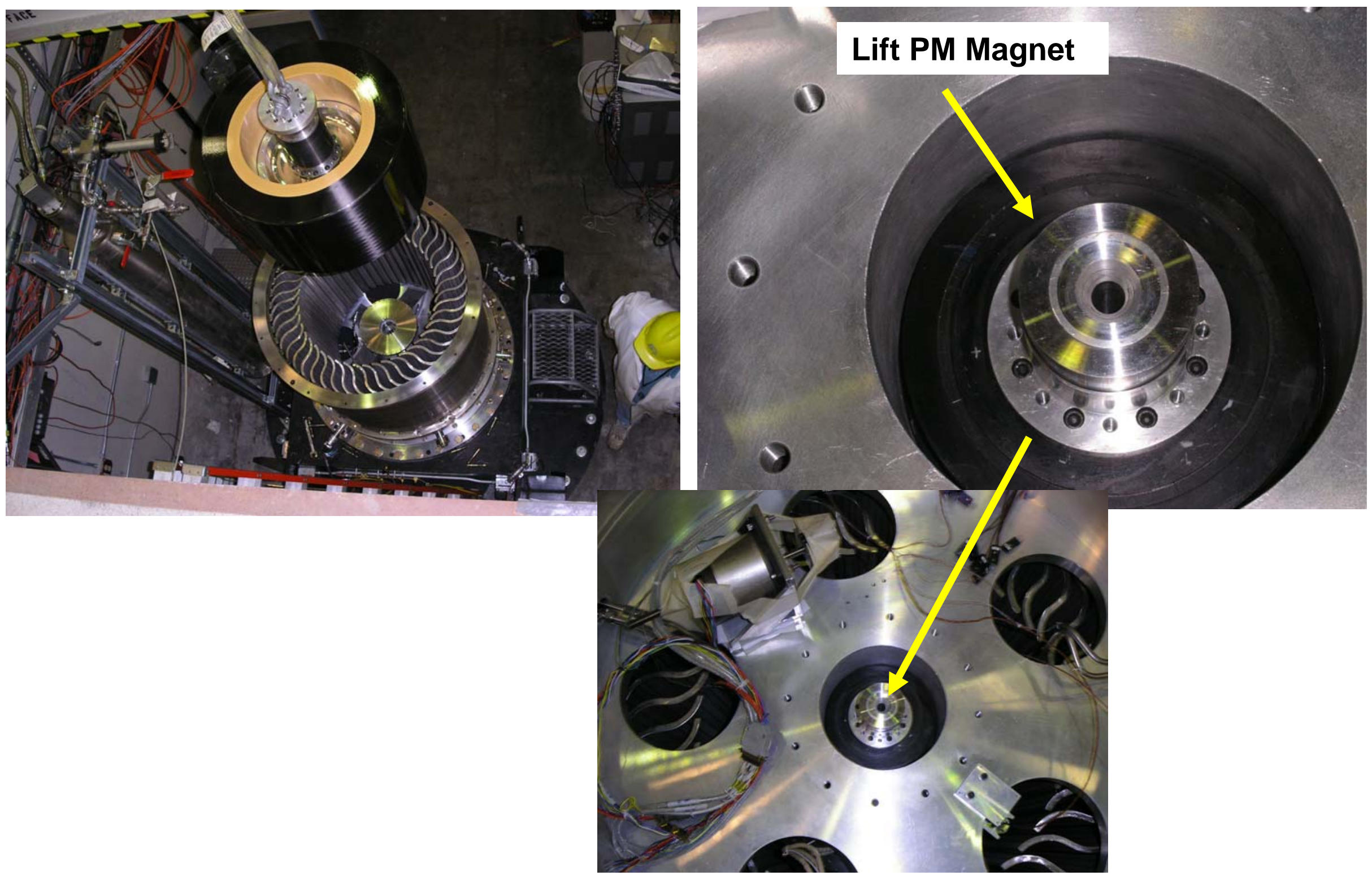




\section{Closing Flywheel Assembly}

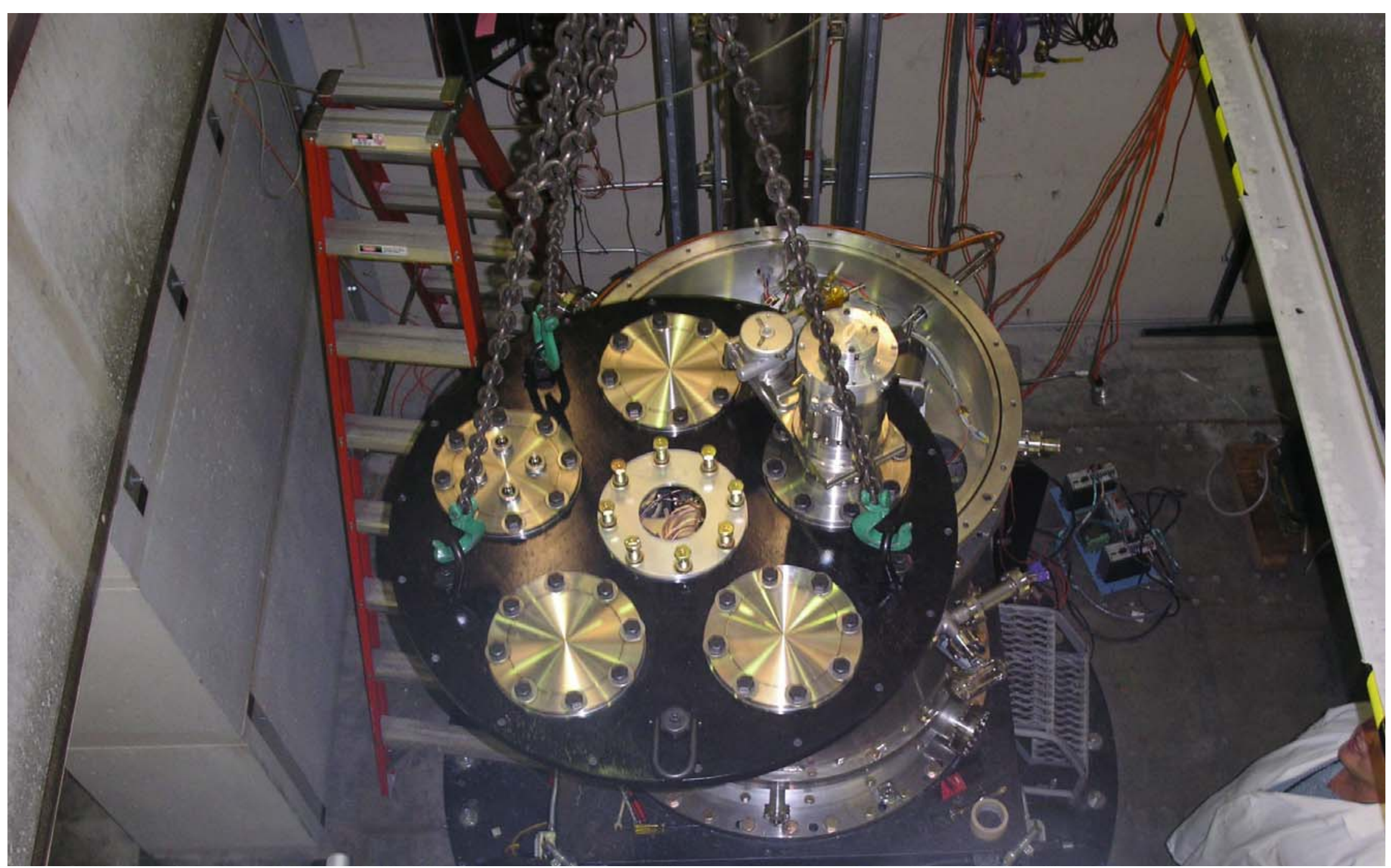




\section{0 kW Power Electronics}

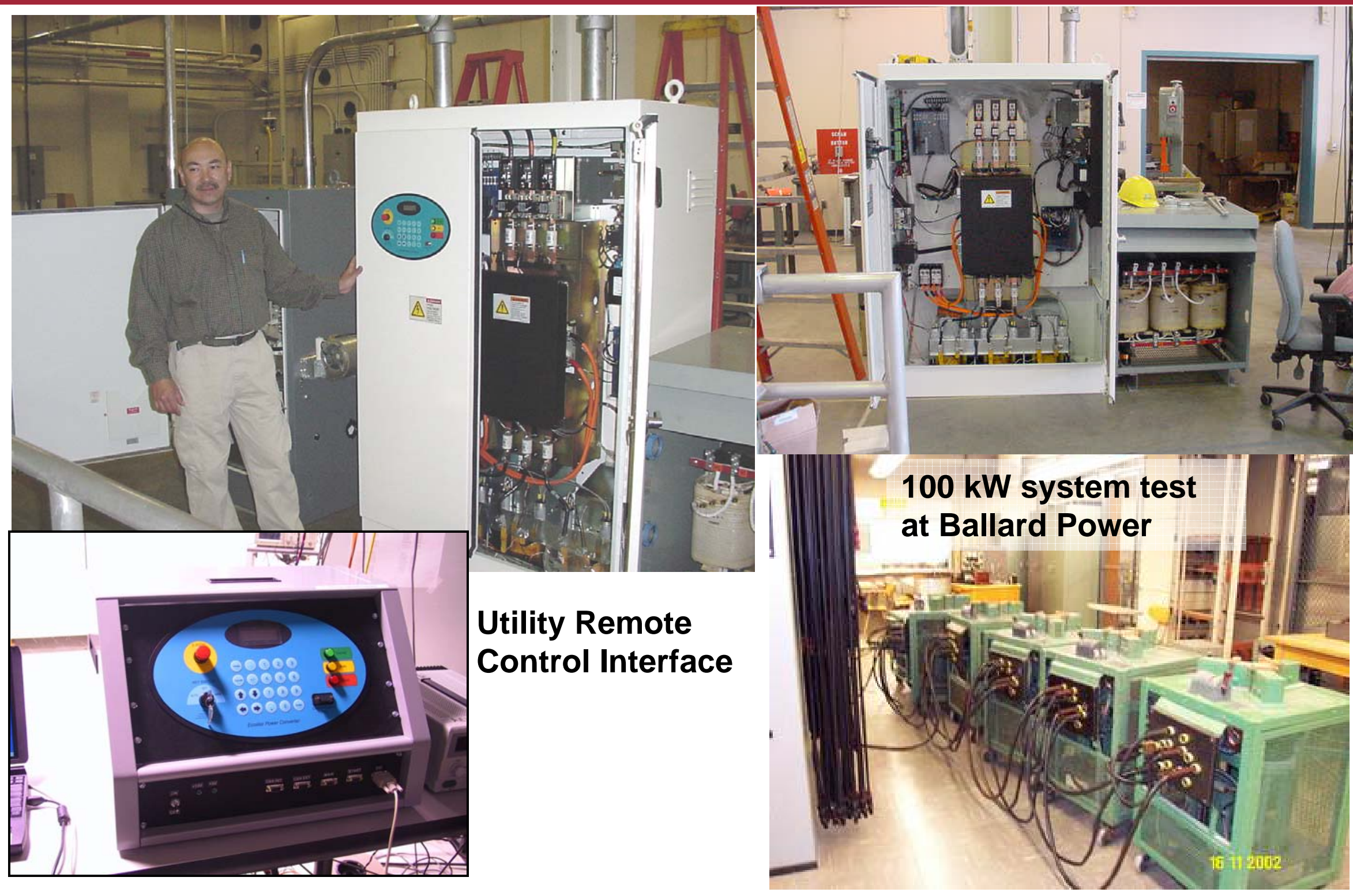




\section{Containment Structure for Rotor Drop/Burst - Subscale Test (after)}

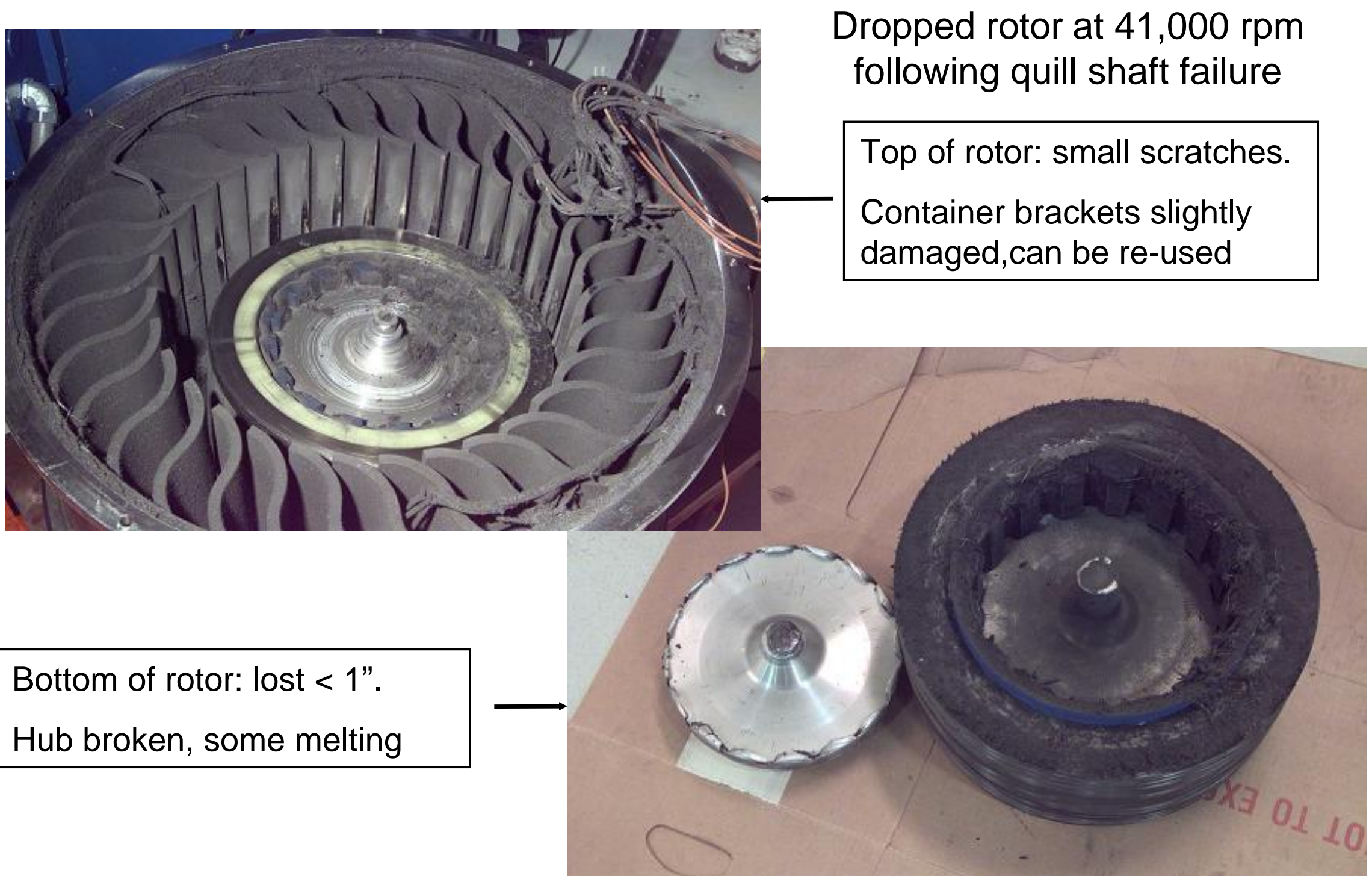


1 kWh Burst Rotor \& Container Before/After

Boeing Technology | Phantom Works

Flywheel Energy Storage

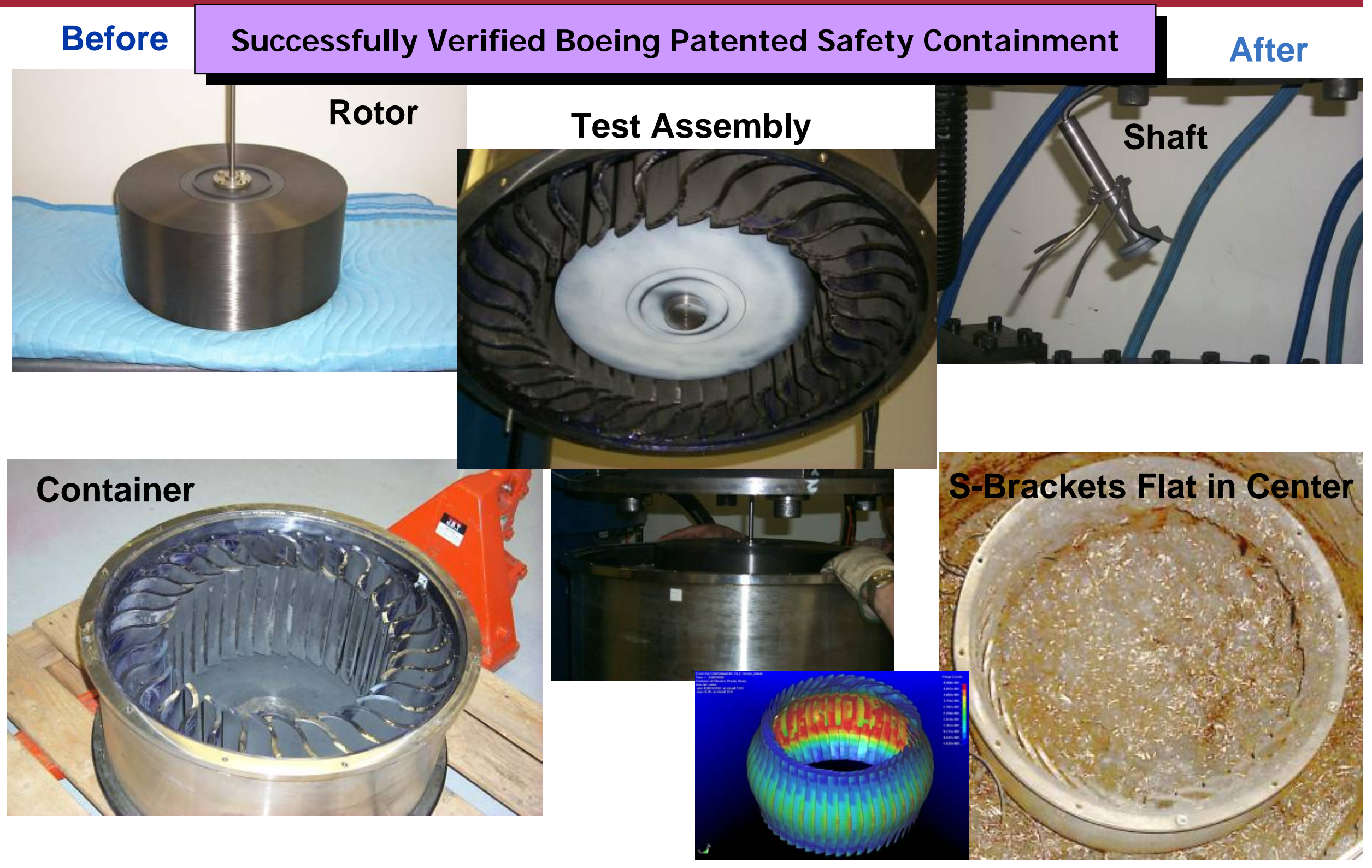




\section{Results of High Speed Touch Down Event}

Boeing Technology | Phantom Works

Flywheel Energy Storage

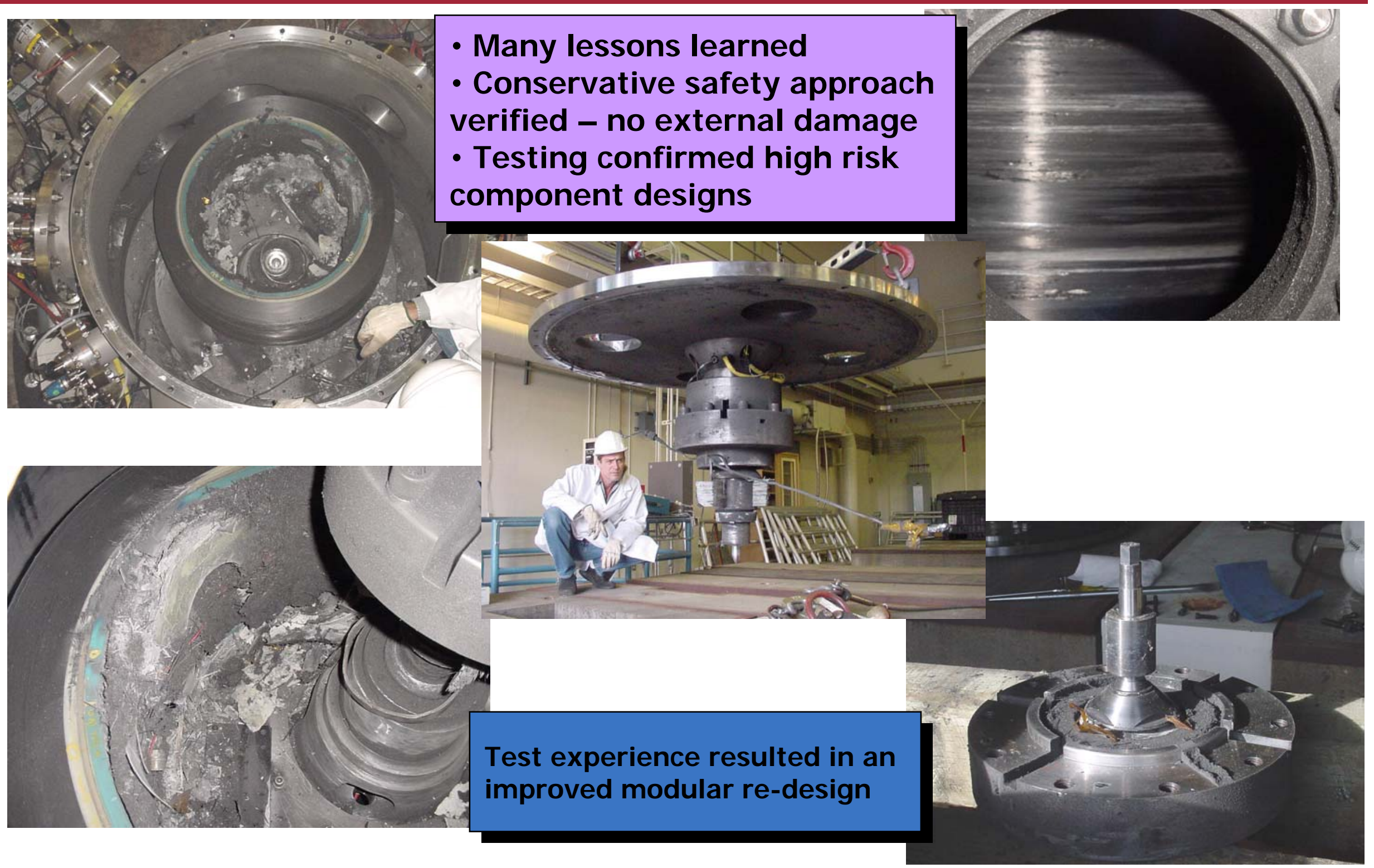




\section{5 kWh/100 kW UPS Flywheel Technical Issues}

Boeing Technology | Phantom Works

Flywheel Energy Storage

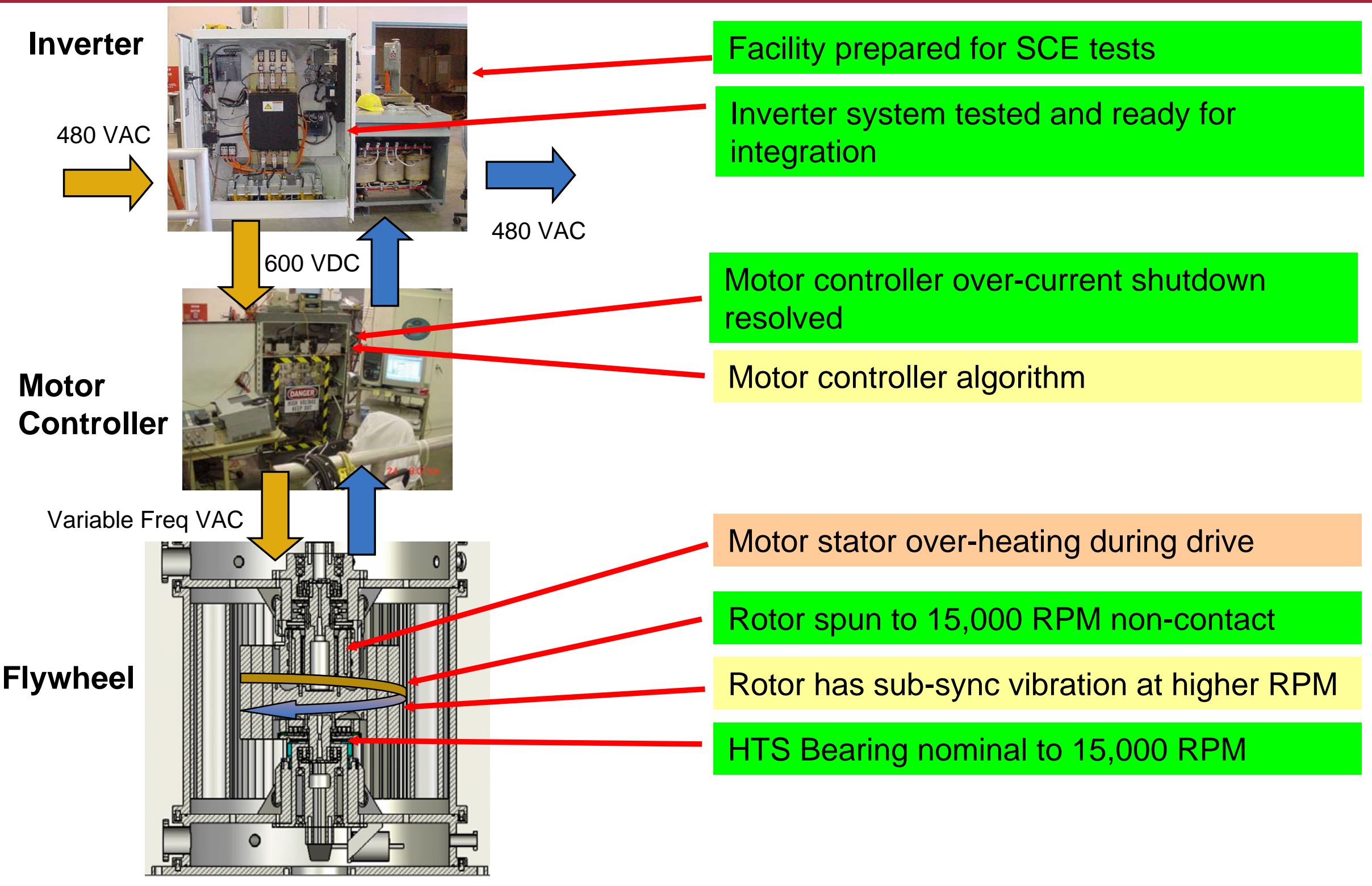




\section{Sub-sync Whirl}

Boeing Technology | Phantom Works

Flywheel Energy Storage
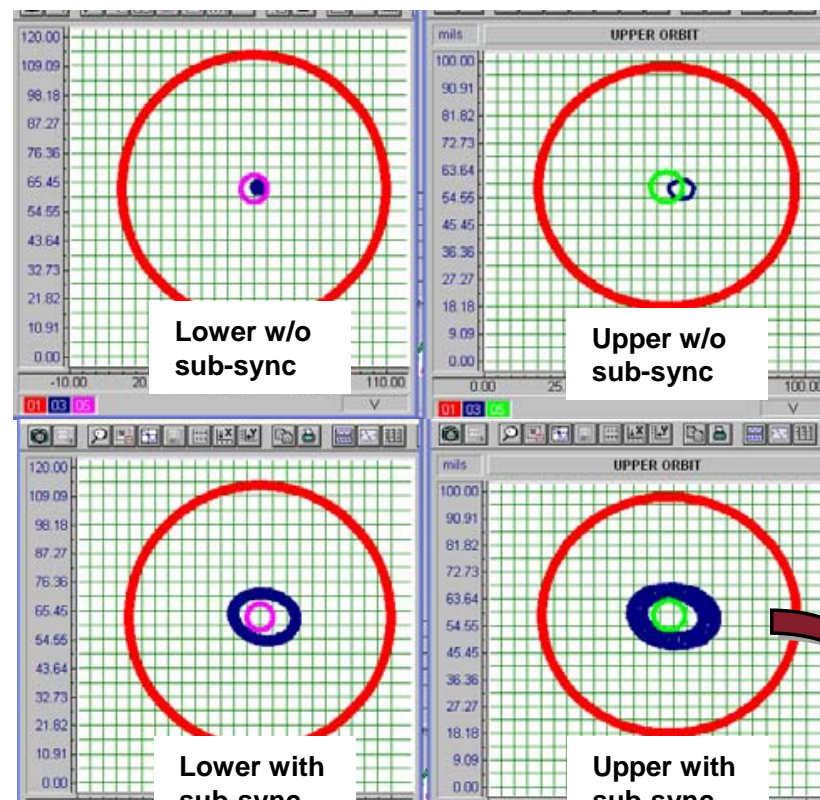

sub-sync

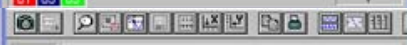

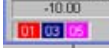
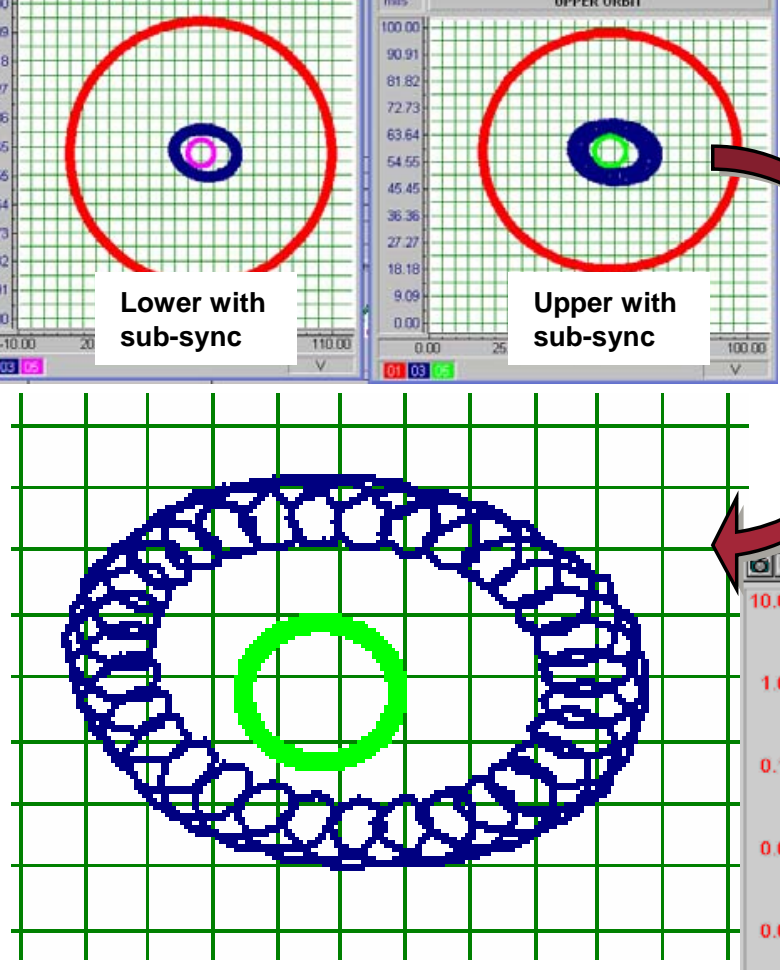

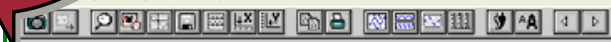

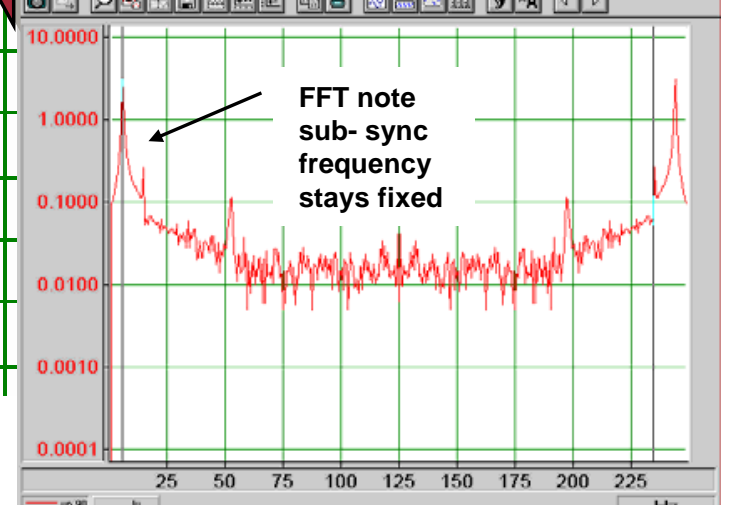

Dec 7, 2005 Run \#5

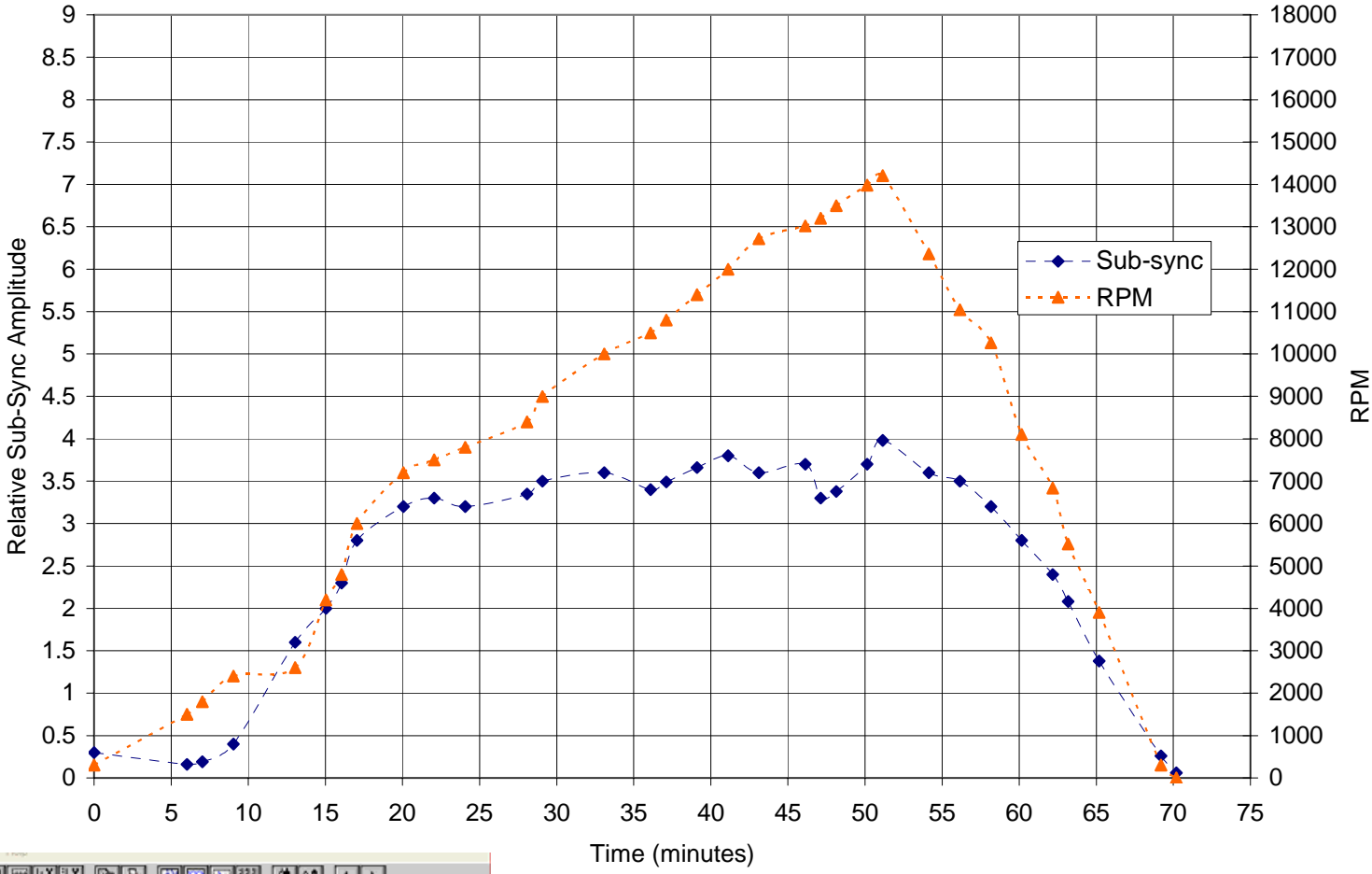

Low energy in sub-sync, but do not want to contact excursion surface 


\section{Proposed System Architecture for Deployment of a 50kW / 5kWh Flywheel Energy Storage System}

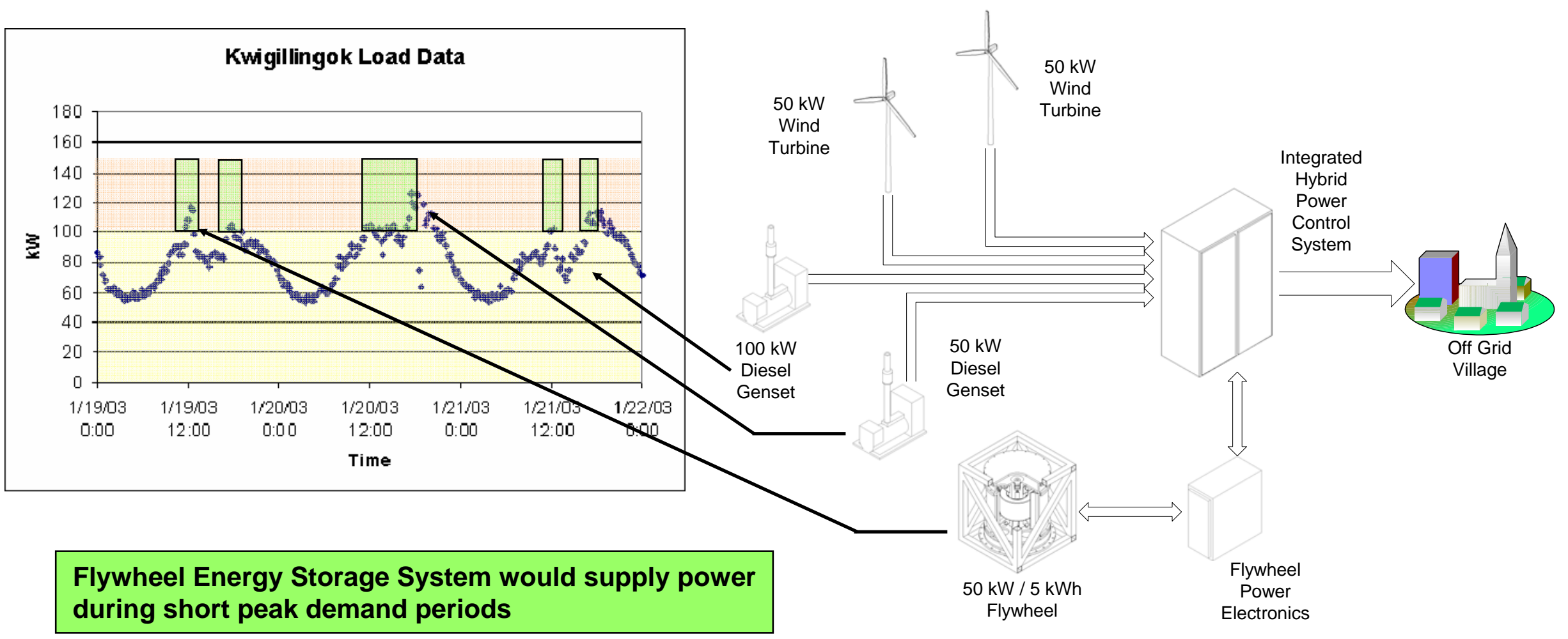

Benefits of Using FESS Instead of Idling $2^{\text {nd }}$ Generator on Standby

- Reduce Generator Maintenance by $\mathbf{5 0 \%}$ (estimate)

- Reduce Fuel Costs by \$80k/yr (estimate)

- Lower Pollution 


\section{Key Issues for HTS Bearing Design}

- Overall efficiency needs to be $>95 \%$ in operating range

- Low loss superconducting bearing

- No criticals in operating range

- System needs to be stiff enough to follow disturbances, yet not so stiff critical frequencies are produced in the operating range

- Interactions between rotating portions (hub, spokes, \& rotor)

- Bearing stiffness

- M/G stiffness

- HTS damping $-J_{c}$ and temperature dependent

- Cooling type - parasitic losses, temperature

- HTS samples size and superconducting properties 


\section{Previous DOE/Boeing Flywheel Terrestrial Cryogenics}

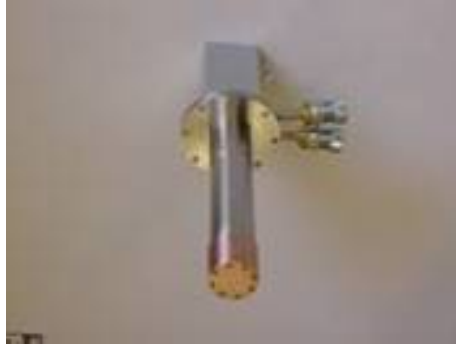

Use of a Thermosiphon eliminated a cryogenic pump requirement

Cold Head (to re-condense $\mathrm{N}_{2}$ gas for closed loop $\mathrm{LN}_{2}$ Operation) \& Liter Size $\mathrm{LN}_{2}$ Reservoir
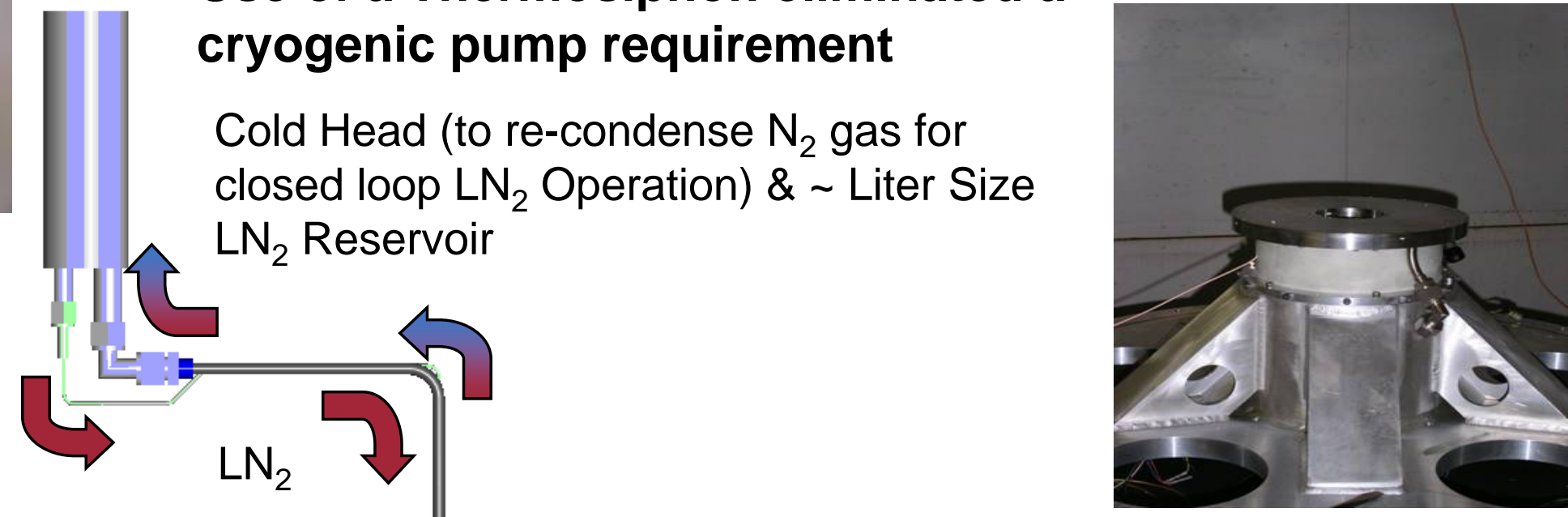

HTS Stability Bearing Cryostat Installed in DOE 5 kWh Flywheel

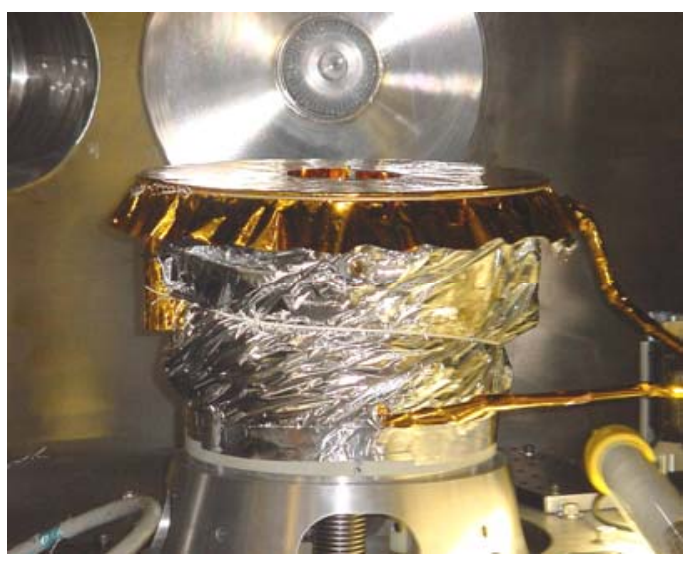

HTS Stability Bearing Cryostat Installed in DOE 10 kWh Flywheel
Return $\mathrm{LN}_{2} \& \mathrm{~N}_{2}$ (Two phase flow)

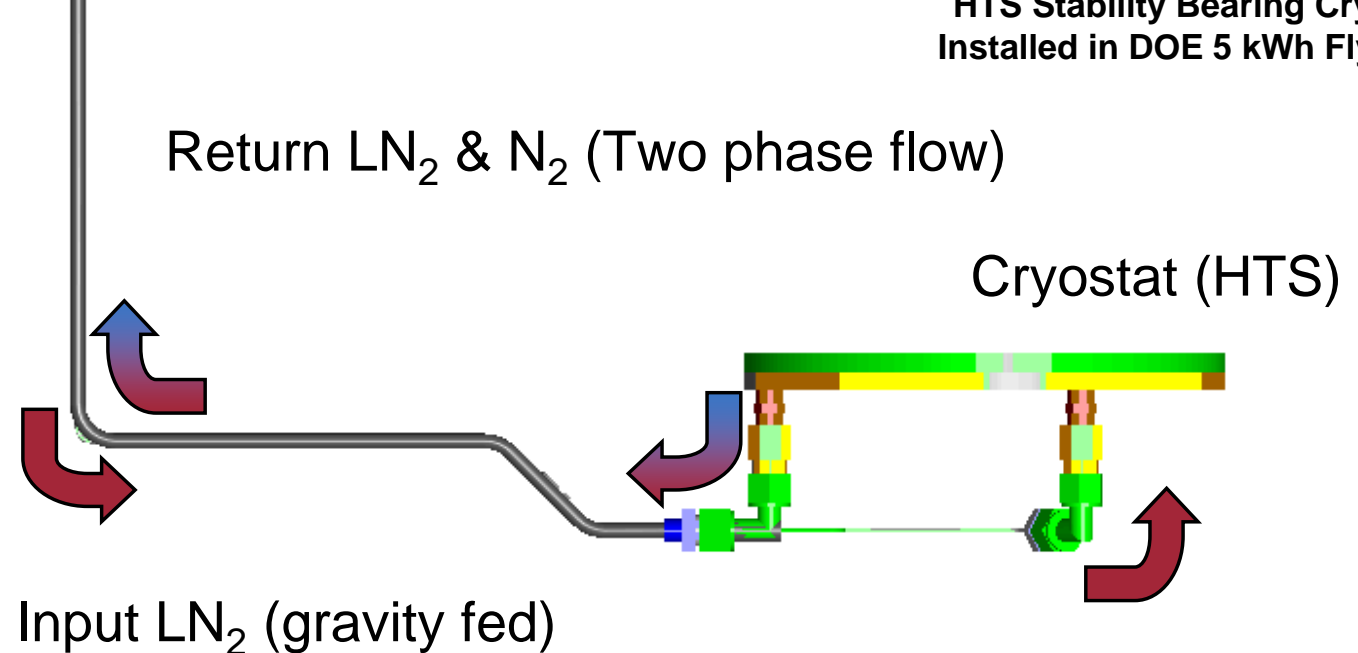

Input $\mathrm{LN}_{2}$ (gravity fed) 


\section{Direct Cooled HTS Bearing}

Boeing Technology | Phantom Works
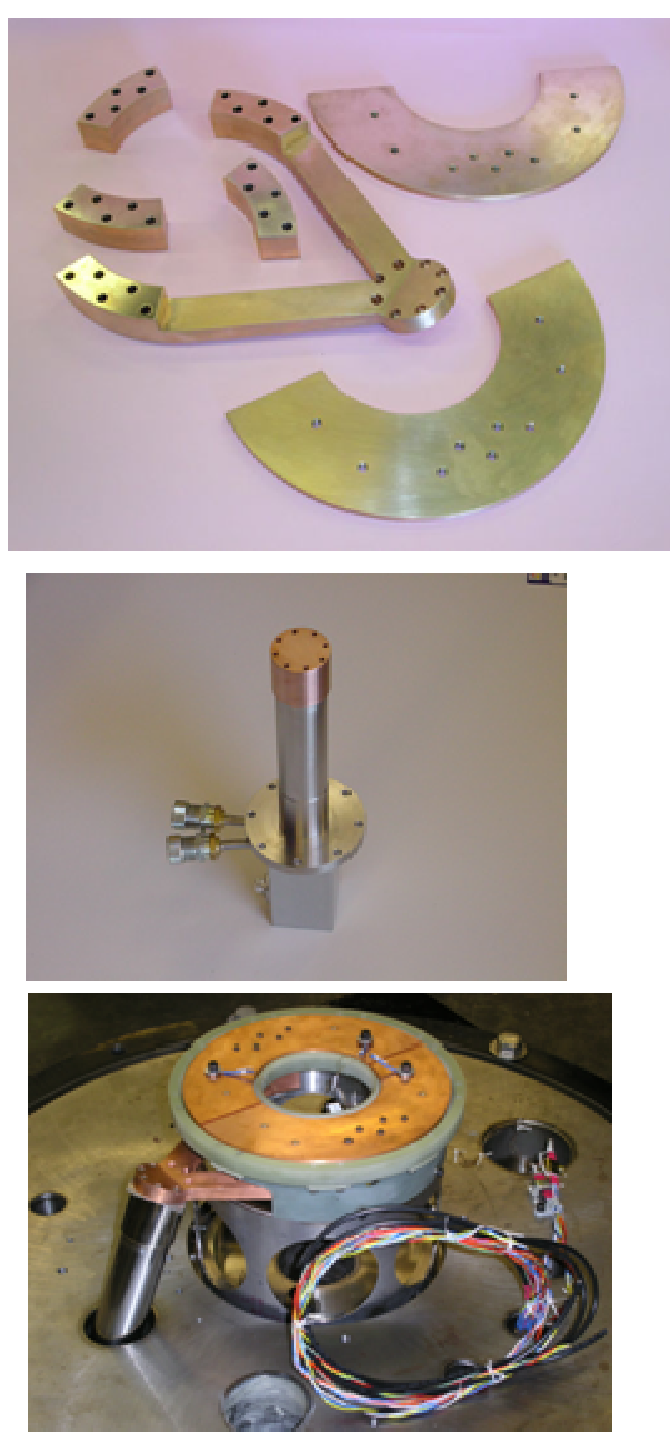

Flywheel Energy Storage
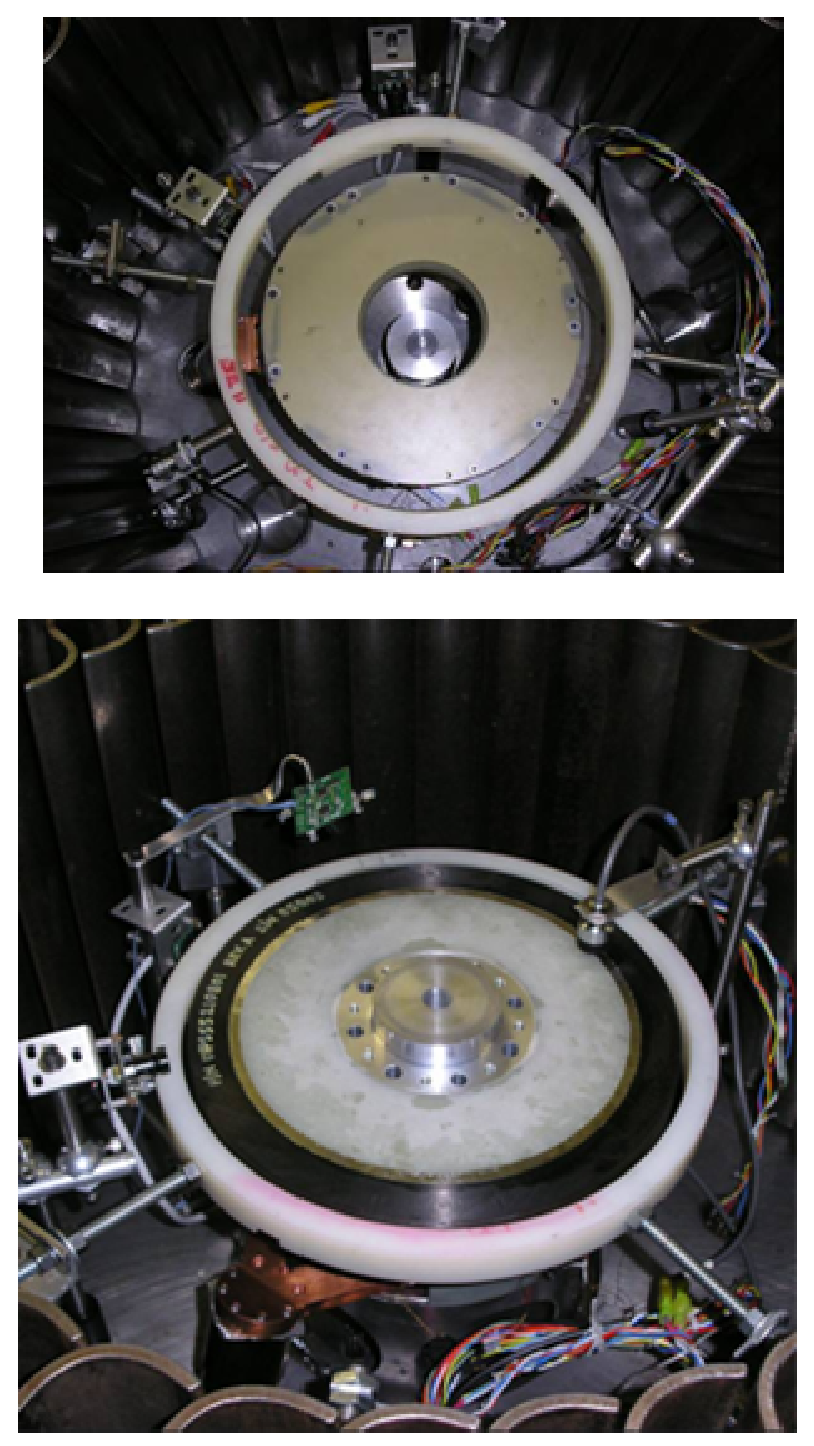
Sandia 50 kW I 5kWh Flywheel Energy Storage System 2007 Direct Cooled Bearing Tests
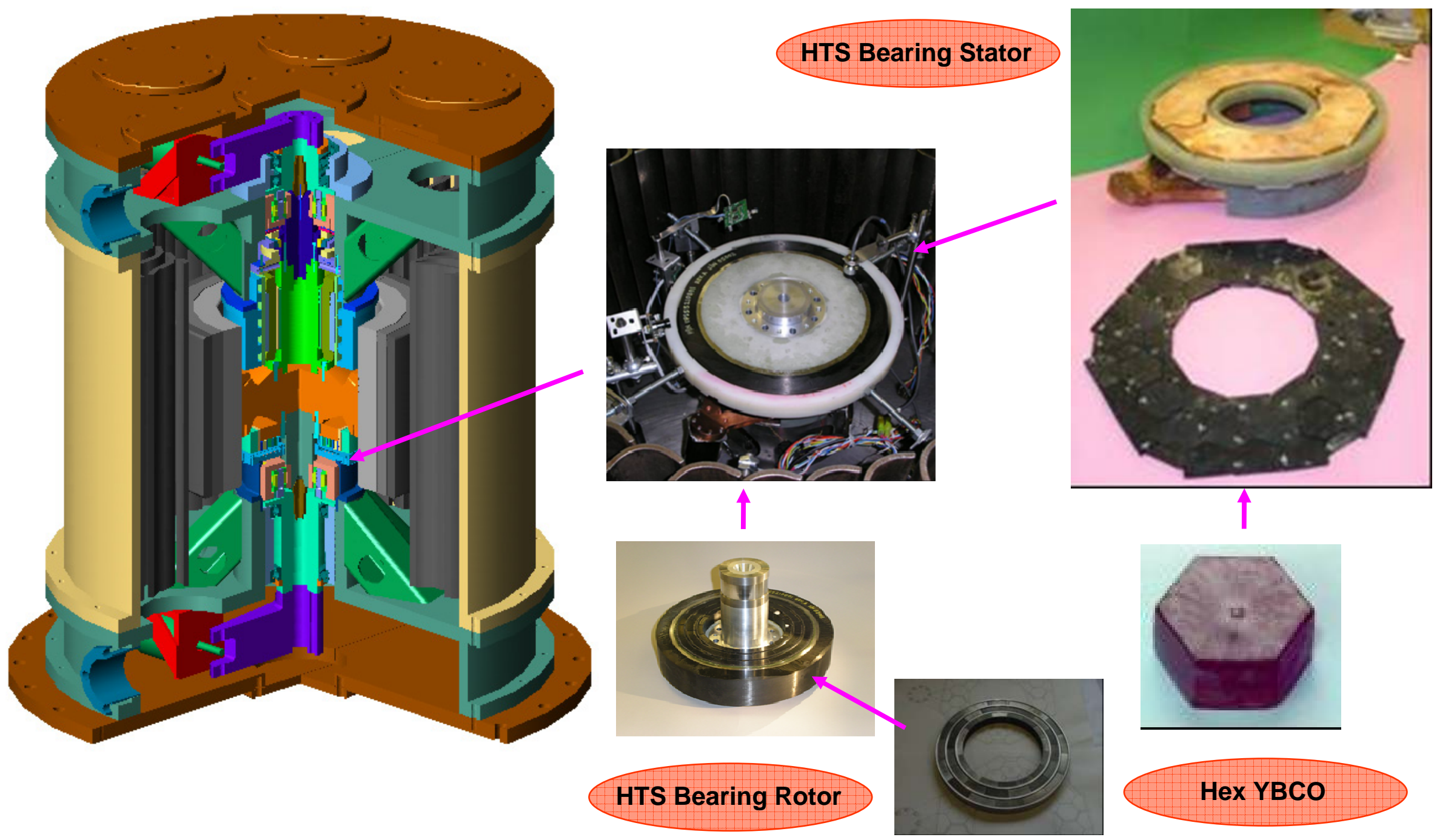

Hex YBCO 


\section{G-10 Bearing Support}

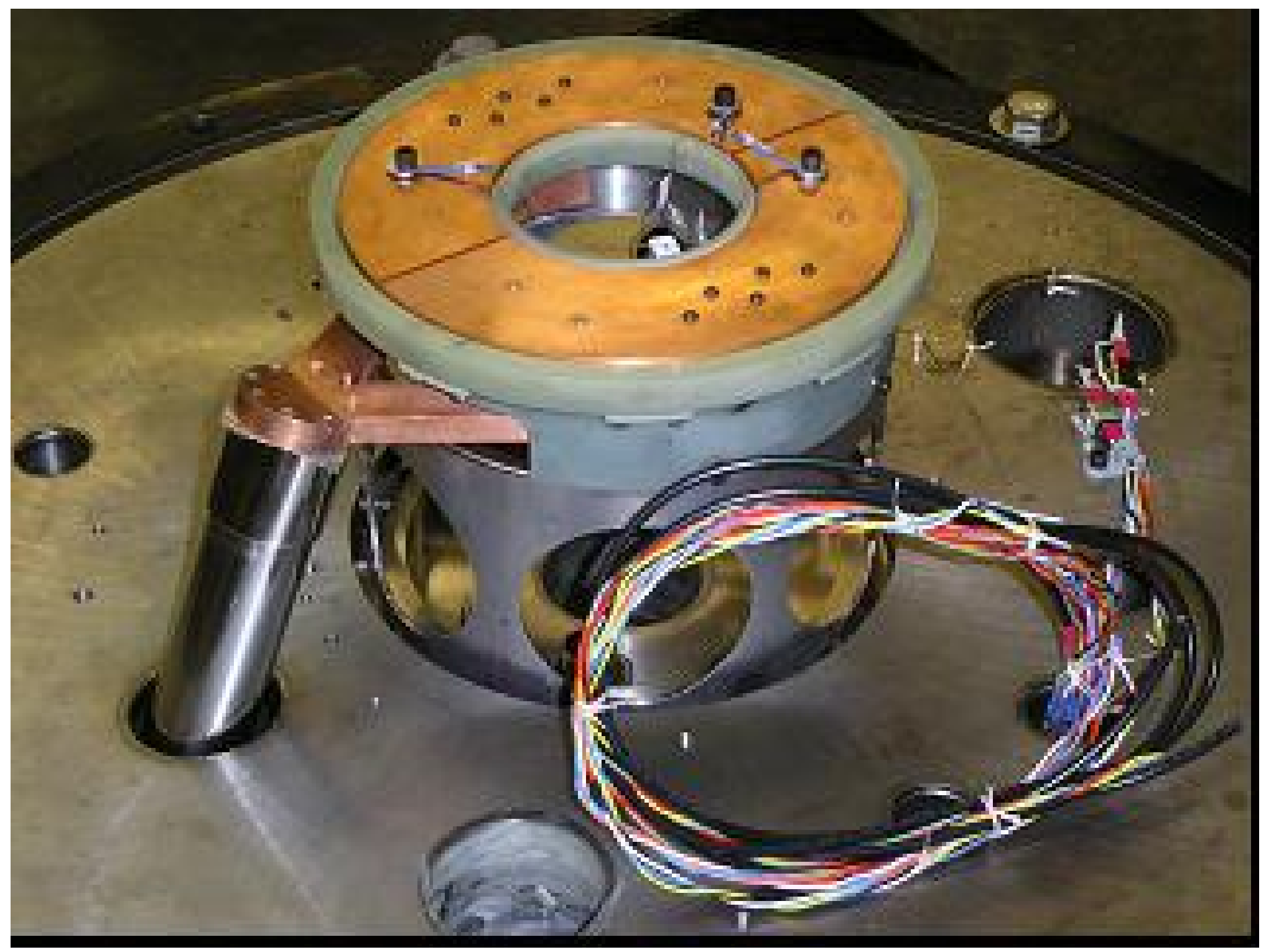




\section{Experimental Spin Down Results from Direct Cooled HTS Bearing}

Boeing Technology | Phantom Works

Flywheel Energy Storage

$\mathrm{T}=50 \mathrm{~K}, \mathrm{Gap}=2.1 \mathrm{~mm}$

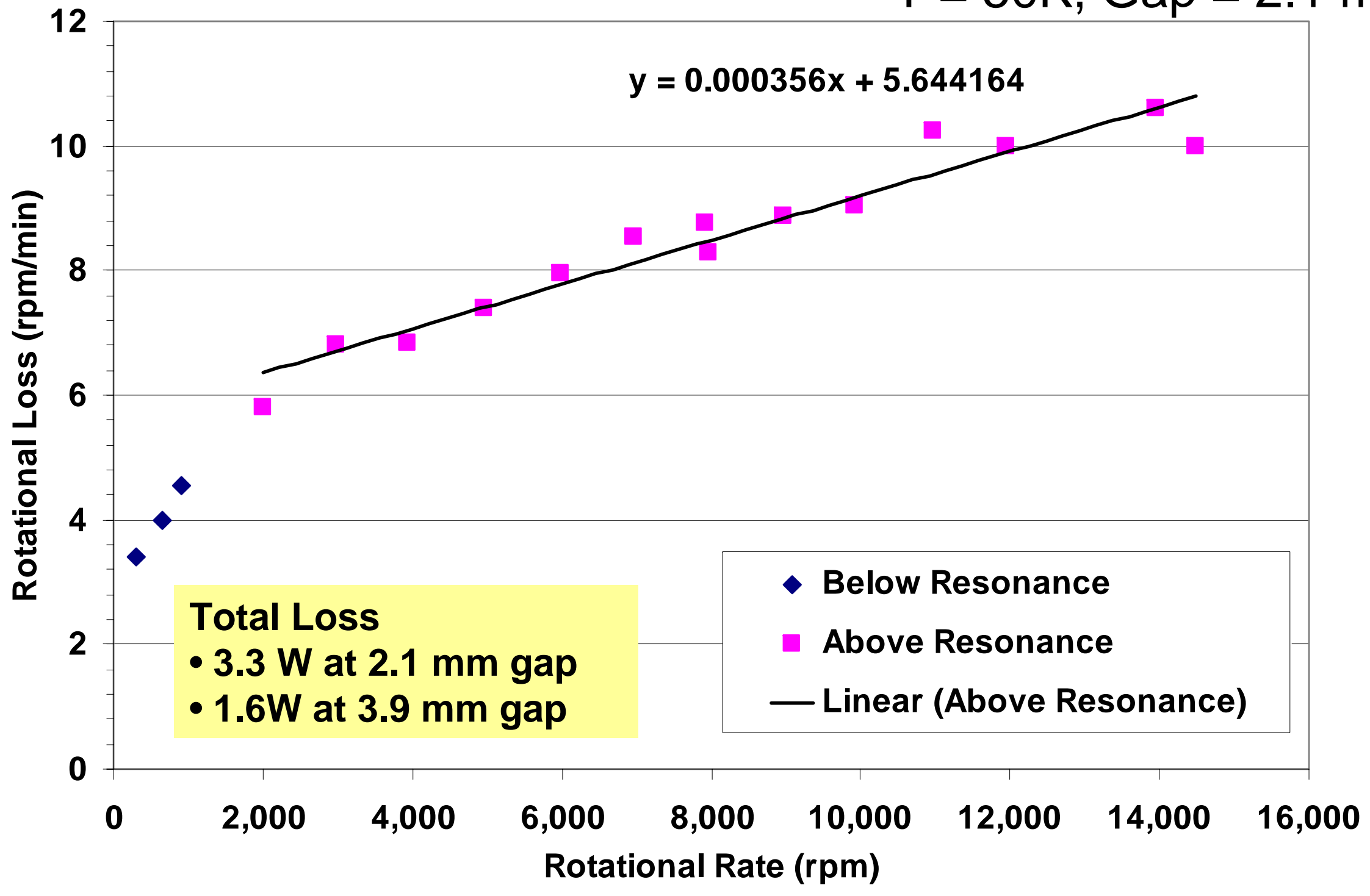




\section{Boeing Flywheel Project Summary}

- Program goal is to design, develop, and demonstrate a $100 \mathrm{~kW}$ UPS flywheel electricity system

- Flywheel system spin tested up to 15,000 RPM in a sensorless, closed loop mode

- Testing identified a manufacturing deficiency in the motor stator - overheats at high speed, limiting maximum power capability

- Successfully spin tested direct cooled HTS bearing up to 14,500 RPM (limited by Eddy current clutch set-up)

- Testing confirmed commercial feasibility of this bearing design - Eddy Current losses are within acceptable limits

- Boeing's investment in flywheel test facilities increased our spin-test capabilities to one of the highest in the nation 